DRAFT VERSiON OCTOBER 15, 2018

Preprint typeset using $\mathrm{IAT}_{\mathrm{E}} \mathrm{X}$ style emulateapj v. 5/2/11

\title{
ON THE JITTER RADIATION
}

\author{
S.R. KELNER \\ National Research Nuclear University (MEPHI), Kashirskoe shosse 31, 115409 Moscow, Russia; Max-Planck-Institut für Kernphysik, \\ Saupfercheckweg 1, D-6917 Heidelberg, Germany
}

F.A. Aharonian

Dublin Institute for Advanced Studies, 31 Fitzwilliam Place, Dublin 2, Ireland; Max-Planck-Institut für Kernphysik, Saupfercheckweg 1, D-6917 Heidelberg, Germany

\section{Khangulyan}

Institute of Space and Astronautical Science/JAXA 3-1-1 Yoshinodai, Chuo-ku, Sagamihara, Kanagawa 252-5210, Japan Draft version October 15, 2018

\begin{abstract}
In a small scale turbulent medium, when the nonrelativistic Larmor radius $R_{\mathrm{L}}=m c^{2} / e B$ exceeds the correlation length $\lambda$ of the magnetic field, the magnetic bremsstrahlung of charged relativistic particles unavoidably proceeds in the so-called jitter radiation regime. The cooling timescale of parent particles is identical to the synchrotron cooling time, thus this radiation regime can be produced with very high efficiency in different astrophysical sources characterized by high turbulence. The jitter radiation has distinct spectral features shifted, compared to synchrotron radiation, towards high energies. This makes the jitter mechanism an attractive broad-band gamma-ray production channel which in highly magnetized and turbulent environments can compete or even dominate over other high energy radiation mechanisms. In this paper we present a novel study on spectral properties of the jitter radiation performed within the framework of perturbation theory. The derived general expression for the spectral power of radiation is presented in a compact and convenient for numerical calculations form.
\end{abstract}

\section{INTRODUCTION}

Charged particles moving in electric and magnetic fields experience effective energy losses via radiation. Because of high conductivity, the electric fields in astrophysical plasmas are typically screened, thus the radiation is dominated by interactions with the magnetic field due to the so-called magnetic bresstrahlung. The latter is one of the major nonthermal radiation processes in astrophysics and operates with high efficiency in a large variety of astrophysical environments. In the case of a regular magnetic field or a chaotic field characterized by large scale fluctuations, we deal with the so-called synchrotron radiation. This process and its implications in astrophysics have been studied in great detail (see e.g. Ginzburg \& Syrovatskii 1969; Rybicki \& Lightman 1979). In highly turbulent environments, namely, when the nonrelativistic Larmor radius $R_{\mathrm{L}}=m c^{2} / e B$ does not exceed the char of $\lambda$, the ceed in significantly different regime, which in the as trophysical literature is referred as diffusive synchrotron radiation (Toptygin \& Fleishman 1987, hereafter TF87) or as jitter radiation (Medvedev 2000, hereafter M00). Hereafter we will use the term "jitter".

The spectral features of jitter radiation substantially differ from the synchrotron radiation. While the power of the synchrotron radiation of a monoenergetic particle $P_{\omega}$ is described with a good accuracy as $\omega P_{\omega} \propto$

\footnotetext{
skelnerorambler.ru

Felly Aharonianempi-hd.mpg.de

khangul@astro.1sas.jaxa.jp
}

$\omega^{4 / 3} \exp \left[-\left(\omega / \omega_{c}\right)\right]$, where $\omega_{c}=3 \gamma^{2} e B /(2 m c)$ is the characteristic synchrotron frequency, in the case of jitter radiation the peak is shifted towards higher frequencies by a factor of $a=R_{\mathrm{L}} / \lambda$. Unless the distribution of emitting particles is strictly monodirectional, the power spectrum of jitter radiation below the maximum is flat, i.e. the Spectral Energy Distribution (SED)円 $\omega P_{\omega} \propto \omega$, while beyond the cutoff energy it has a power-law behavior, $\omega P_{\omega} \propto \omega^{1-\alpha}$, where $\alpha$ is the power-law index of the turbulence spectrum (TF87). Thus, instead of the typical exponential cutoff in synchrotron spectrum, the jitter mechanism yields a power-law spectrum which can be extended up to the frequency of $a^{3} \omega_{c}$. This makes the jitter radiation of electrons an excellent high energy gamma-ray production process in contrast to the synchrotron radiation which even in the case of extreme accelerators operating at the maximum possible rate allowed by classical electrodynamics Aharonian et al. 2002) is limited by the maximum possible energy $\epsilon_{0}=\hbar \omega_{0}=9 / 4 \alpha^{-1} m c^{2} \sim 150 \mathrm{MeV}$.

However, so far this remarkable feature of jitter radiation practically has not been explored for interpretation of high energy gamma-ray phenomena (see however, the recent papers by Teraki \& Takahara 2013). Instead, more emphasis has been placed on the energy interval below the cutoff. In particular, it is augured in M00

1 The so-called Spectral Energy Distribution or SED is determined as $\omega^{2} d N / d \omega$ or $\epsilon^{2} d N / d \epsilon$, where $d N / d \omega(d N / d \epsilon)$ is the differential distribution over frequencies (energies). Obviously here the SED is $\omega P_{\omega}$; note that in astronomical literature for SED is often used the denotation $\nu F_{\nu}$. 
that the jitter radiation below the cutoff can result in harder spectra than the synchrotron radiation, namely $\omega P_{\omega} \propto \omega^{2}$. However, the claimed energy dependence is close y related to the assumed geometry of the magnetic field. Namely, it can be achieved if the magnetic field has only one non-zero component which can be realized only for a rather unrealistic configuration of the turbulent field (see Sect. 7 for details).

In the seminal paper on jitter radiation by $\mathrm{TF} 87$ it has been realized that the spectral maximum of the jitter emission is located at higher frequencies than in the synchrotron regime, and that the high energy part of the jitter spectrum could be described by a power-law. Thus, even for the case of monoenergetic particle distribution one may expect a broken power-law spectrum. This should lead to the modification of the standard relations between spectral slopes, flux levels and breaks found in synchrotron spectra. Possible applications of the jitter mechanism also has been discussed, basically in the low energy range of cosmic electromagnetic radiation. In particular, it has been proposed that the jitter radiation can be responsible for the radio to optical (Xray) spectra of some active galaxies and pulsar wind nebulae (TF87:Fleishman \& Bietenholz 2007; Mao \& Wang 2007 )

The underestimation of the potential of jitter radiation for production of high and very high energy gamma-rays could be related to the effect of weakening of the diffusive shock acceleration process in the case of short-length scale turbulence. A self-consistent consideration of the processes of particle acceleration and emission in the framework of the diffusive shock acceleration paradigm predicts a shift of the jitter radiation peak towards low frequencies as compared to the pure synchrotron radiation (Derishev 2007, Kirk \& Reville 2010). However, if the inhomogeneities responsible for particle acceleration and emission are different, e.g. when these processes occur in spatially separated regions, the spectral maximum would be shifted towards higher energies making the jitter radiation a very effective high energy gamma-ray production mechanism. Therefore the spectral features of this radiation in the entire energy range deserve detailed qualitative studies.

To explore the process in a general form, we propose a new approach based on the perturbation theory. In terms of additional assumptions, the proposed method is less demanding compared to previous studies, and allows a precise control of the applicability conditions for the derived solutions, e.g. the range of the high energy power-law extension beyond the spectral maximum.

In this regard we should note that in previous studies some principal results have been obtained under specific, although not always obvious assumptions. For example, M00 has derived the spectrum of radiation for the case of a very specific geometry of the magnetic field fluctuations. In some others studies (see e.g., Fleishman \& Biet nholz 2007) the jitter radiation spectrum in fact has not been strictly derived, but rather predefined through its asymptotic behavior. Finally, some studies address the case of anisotropic turbulence (Reynolds \& Medvedev 2012), however the structure of the used correlation tensor is not consistent with the fundamental requirement of $\nabla \cdot \boldsymbol{B}=0$. We discuss these concerns in detail in Section 7 .
Finally, we should mention that a significant progress recently has been achieved through numerical computations based on particle-in-cell technique (see, e.g., Reville \& Kirk 2010; Teraki \& Takahara 2011). This method has a great potential to deal with quite complex distributions of emitting particles. On the other had, the analytical approach allows better understanding and interpretation of physics behind the obtained results. In this regard, two methods are complementary and equally important.

The paper is organized as following: in Section 2 the basic results on the energy spectra, as well as the applicability limits for the derived spectra are presented. in Section 3 we consider the case of chaotic magnetic field. In Section 5 we compare the radiation properties in chaotic magnetic field with the conventional synchrotron radiation; the latter is is briefly discussed in Section 4 . The case of anisotropic turbulence (under assumption of isotropic distribution of emitting particles) is considered in Section 6. Finally, we compare our results with previous studies in Section 7, and summarize the main results in Section 8 .

\section{PERTURBATION THEORY}

The intensity and the energy distribution of radiation produced by a particle of a given charge $e$ depends only on its trajectory. Let $\boldsymbol{r}(t)$ and $\boldsymbol{v}(t)=\dot{\boldsymbol{r}}(t)$ be the radiusvector and the velocity of the particle at the instant $t$. Then, the energy spectrum of radiation is described by equation (14.65) of Jackson (1998) which for our purposes is convenient to present in the form 2

$$
\begin{aligned}
\frac{d \mathscr{E}_{\boldsymbol{n} \omega}}{d \omega d \Omega} & =\frac{e^{2}}{4 \pi^{2} c^{3}}\left|\int_{\mathbb{R}} \boldsymbol{U}(t) d t\right|^{2} \\
& =\frac{e^{2}}{4 \pi^{2} c^{3}} \int_{\mathbb{R}^{2}} \boldsymbol{U}\left(t_{1}\right) \boldsymbol{U}^{*}\left(t_{2}\right) d t_{1} d t_{2} .
\end{aligned}
$$

Here the integrand

$$
\boldsymbol{U}(t)=\frac{\boldsymbol{n} \times[(\boldsymbol{n}-\boldsymbol{\beta}(t)) \times \boldsymbol{a}(t)]}{(1-\boldsymbol{n} \boldsymbol{\beta}(t))^{2}} e^{i \Phi(t)}
$$

depends on the particle velocity $\boldsymbol{v}(t)=c \boldsymbol{\beta}(t)$ and the acceleration $\boldsymbol{a}(t)=c \dot{\boldsymbol{\beta}}(t)$, as well as the function $\Phi(t)=$ $\omega(t-\boldsymbol{n} \boldsymbol{r}(t) / c)$, where $\boldsymbol{n}$ is the unit vector towards the momentum of the radiated photon. The function $\boldsymbol{U}^{*}(t)$ is the is complex conjugation of $\boldsymbol{U}(t)$. Note that equation (11) is precise; it is derived within the framework of classical electrodynamics through integration of the Maxwell equations in vacuum.

It is convenient to introduce new variables of integration: $t=\left(t_{1}+t_{2}\right) / 2$ and $\tau=t_{1}-t_{2}$ (note that $\left.d t_{1} d t_{2}=d t d \tau\right)$. Then equation (11) results in

$$
\frac{d \mathscr{E}_{\boldsymbol{n} \omega}}{d \omega d \Omega}=\frac{e^{2}}{4 \pi^{2} c^{3}} \int \boldsymbol{U}(t+\tau / 2) \boldsymbol{U}^{*}(t-\tau / 2) d t d \tau .
$$

As it is shown bellow, for a fixed value of $t$ the integrand rapidly decreases with the increase of $|\tau|$. Also, the integrand is characterized by a weak dependence on $t$. The

${ }^{2} \mathrm{~d} \mathscr{E}$ n $\omega$ is the energy radiated by a particle into the solid angle $\mathrm{d} \Omega$ within the frequency interval $\mathrm{d} \omega$. 
integration of the integrand over $\mathrm{d} \tau$ gives the spectral power of emission at the moment $t$ :

$$
P_{\boldsymbol{n} \omega}(t)=\frac{e^{2}}{4 \pi^{2} c^{3}} \int_{-\infty}^{\infty} \boldsymbol{U}(t+\tau / 2) \boldsymbol{U}^{*}(t-\tau / 2) d \tau .
$$

Note that if one considers equation (3) as a classical limit of the corresponding quantum relation, then the integrand in equation (3) can be interpreted as the emission probability multiplied to the photon energy $\hbar \omega$ (see Berestetskii et al. 1989, § 90).

The radiation detected by an observer is produced by ensemble of particles occupying a certain region in space. We will consider the case of a chaotic magnetic field, assuming that statistically averaged (over time or space) magnetic field $\langle\boldsymbol{B}\rangle=0$.

Let $\lambda$ be the correlation length of the magnetic field. If the distance between two chosen points at $\boldsymbol{r}_{1}$ and $\boldsymbol{r}_{2}$ exceeds $\lambda$, then the corresponding magnetic fields $\boldsymbol{B}_{1}$ and $\boldsymbol{B}_{2}$ can be treated as statistically independent, thus the time-averaged product of these fields $\left\langle B_{1 \rho} B_{2 \sigma}\right\rangle=$ $\left\langle B_{1 \rho}\right\rangle\left\langle B_{2 \sigma}\right\rangle=0$.

To obtain the radiation spectrum, the integrand in equation (11) should be averaged over all possible configurations of the magnetic field. It is convenient to perform this procedure in the framework of perturbation theory. The acceleration of particle is proportional to the strength of the magnetic field $\boldsymbol{B}, \boldsymbol{a}=e(\boldsymbol{\beta} \times \boldsymbol{B}) /(m \gamma)$. In the first approximation, all other relevant parameters can be treated as in the absence of the magnetic field, i.e. $\boldsymbol{\beta}(t \pm \tau / 2)=\boldsymbol{\beta}(t), \boldsymbol{r}(t \pm \tau / 2)=\boldsymbol{r}(t) \pm \boldsymbol{\beta}(t) \tau / 2$. The applicability of the approach is discussed bellow. This approximation results in

$$
\begin{gathered}
P_{\boldsymbol{n} \omega}(t)=\frac{e^{2}}{4 \pi^{2} c^{3}(1-\boldsymbol{n} \boldsymbol{\beta})^{2}} \\
\times \int_{-\infty}^{\infty}\left[\boldsymbol{a}_{+} \boldsymbol{a}_{-}-\frac{\left(\boldsymbol{n} \boldsymbol{a}_{+}\right)\left(\boldsymbol{n} \boldsymbol{a}_{-}\right)}{\gamma^{2}(1-\boldsymbol{n} \boldsymbol{\beta})^{2}}\right] e^{i \omega(1-\boldsymbol{n} \boldsymbol{\beta}) \tau} d \tau,
\end{gathered}
$$

where $\boldsymbol{a}_{ \pm}=\boldsymbol{a}(t \pm \tau / 2)$ and $\left.\boldsymbol{\beta}=\boldsymbol{\beta}(t)\right) ; \gamma=1 / \sqrt{1-\beta^{2}}$ is the particle Lorentz factor. The derivation of equation (5) was performed using the formula for the standard double vector product: $\boldsymbol{a} \times(\boldsymbol{b} \times \boldsymbol{c})=\boldsymbol{b}(\boldsymbol{a} \boldsymbol{c})-\boldsymbol{c}(\boldsymbol{a} \boldsymbol{b})$ and taking into account that in the magnetic field the acceleration and velocity vectors are orthogonal. Equation (5) represents the first non-vanishing term in the expansion of the emission spectrum in powers of the magnetic field.

Our ultimate aim is to derive the emission spectrum integrated over the emission angles of photons and averaged over the magnetic field fluctuations. It is convenient to select the $z$ axis to be parallel to the particle velocity $\boldsymbol{\beta}$, and start with averaging over the azimuthal angle $\phi$ in respect to the direction of the particle velocity $\boldsymbol{\beta}$. Then, the scalar product of the vectors $\boldsymbol{n}$ and $\boldsymbol{\beta}$ does not depend on the azimuthal angle $\phi,(\boldsymbol{n} \boldsymbol{\beta})=\beta \cos \theta$. Given that $\boldsymbol{a}_{ \pm} \perp \boldsymbol{\beta}$, one obtains

$$
\left\langle\left(\boldsymbol{n} \boldsymbol{a}_{+}\right)\left(\boldsymbol{n} \boldsymbol{a}_{-}\right)\right\rangle=\frac{1}{2}\left(\boldsymbol{a}_{+} \boldsymbol{a}_{-}\right) \sin ^{2} \theta .
$$

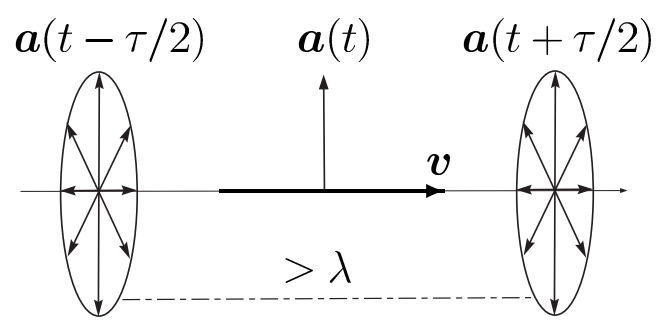

FIG. 1.- Schematic description of the basic geometry adopted for computations. Thin solid line represents a segment of the trajectory of the charge particle, which in the framework of the perturbation approach can be taken as a straight line. The particle acceleration is orthogonal to the velocity (which in its turn is parallel to the trajectory). Also it is assumed that the acceleration is statistically independent for distances along the trajectory which exceed the magnetic field correlation length $\lambda$. This corresponds to the condition $|\tau|>\lambda / c$.

and, after averaging of equation (5) over $\phi$, we have

$$
\begin{aligned}
P_{\boldsymbol{n} \omega}(t)= & \frac{e^{2}}{4 \pi^{2} c^{3}(1-\boldsymbol{n} \boldsymbol{\beta})^{2}}\left(1-\frac{\sin ^{2} \theta}{2 \gamma^{2}(1-\boldsymbol{n} \boldsymbol{\beta})^{2}}\right) \\
& \times \int_{-\infty}^{\infty}\left(\boldsymbol{a}_{+} \boldsymbol{a}_{-}\right) e^{i \omega(1-\boldsymbol{n} \boldsymbol{\beta}) \tau} d \tau .
\end{aligned}
$$

In equation (7) the charge velocity, $\boldsymbol{\beta}=\boldsymbol{\beta}(t)$, is treated as a constant (independent of $\tau$ ). The averaging over the magnetic field configurations results in appearance of a correlation function, $\left\langle\boldsymbol{a}_{+} a_{-}\right\rangle$, under the integral. Note that for $\boldsymbol{\beta}=$ const, the acceleration and magnetic field have the same statistical properties. In particular, $\langle\boldsymbol{a}(t)\rangle=0$ and $\langle\boldsymbol{a}(t+\tau / 2) \boldsymbol{a}(t-\tau / 2)\rangle=$ $\langle\boldsymbol{a}(t+\tau / 2)\rangle\langle\boldsymbol{a}(t-\tau / 2)\rangle=0$ if the distance between the corresponding points exceeds $\lambda$ (i.e., if $c \beta \tau>\lambda$ ). This feature of $\left\langle\boldsymbol{a}_{+} a_{-}\right\rangle$is illustrated in figure 1 .

Since the radiation of ultrarelativistic particles is strongly beamed towards the direction of motion $(\theta \sim$ $1 / \gamma)$, we will consider only the region of small angles, $\theta \ll 1$. This allows significant simplifications of calculations which result in

$$
P_{\boldsymbol{n} \omega}(t)=\frac{e^{2}}{\pi^{2} c^{3}} \frac{\gamma^{4}\left(1+\gamma^{4} \theta^{4}\right)}{\left(1+\gamma^{2} \theta^{2}\right)^{4}} \int_{-\infty}^{\infty}\left\langle\boldsymbol{a}_{+} \boldsymbol{a}_{-}\right\rangle e^{i \tilde{\omega} \tau} d \tau,
$$

where

$$
\tilde{\omega}=\frac{\omega}{2 \gamma^{2}}\left(1+\gamma^{2} \theta^{2}\right) .
$$

Since the above results are derived within the framework of the perturbation approach, it is important to study the range of applicability of equation (8).

The integrand in equation (8) rapidly decreases in the range of $|\tau| \gtrsim \lambda / c$. Therefore, the obtained expression describes correctly the emission power if the terms neglected at derivation of equation (8) are small for $|\tau| \lesssim \lambda / c$. In the precise equation (雨), the denominator contains a term $d_{ \pm} \equiv 1-\boldsymbol{n} \boldsymbol{\beta}(t \pm \tau / 2)$. For small values of $\tau$, we have $d_{ \pm}=(1-\boldsymbol{n} \boldsymbol{\beta}) \mp \boldsymbol{n} \dot{\boldsymbol{\beta}} \frac{\tau}{2}$. For ultrarelativistic particles the angle $\theta$ between $\boldsymbol{n}$ and $\boldsymbol{\beta}$ is small $(\sim 1 / \gamma)$, thus, given the orthogonality of $\dot{\boldsymbol{\beta}}$ and $\boldsymbol{\beta}$, the last term in $d_{ \pm}$can be estimated as $\frac{e B \lambda}{m c^{2} \gamma^{2}}$. Since this term was neglected at derivation of equation (8), it must be small compared to the first term in $d_{ \pm}$, which 
is estimated as $\sim 1 / \gamma^{2}$. Thus, the range of applicability is determined by the condition

$$
\frac{e B \lambda}{m c^{2}} \ll 1
$$

Furthermore, the exponential term in equation (14) contains a function, $i \Delta \equiv i(\Phi(t+\tau / 2)-\Phi(t-\tau / 2))$. The Taylor expansion of the function $\Delta$ gives $\Delta=\omega(1-$ $\boldsymbol{n} \boldsymbol{\beta}) \tau-\omega \boldsymbol{n} \ddot{\boldsymbol{\beta}} \tau^{3} / 24$. In derivation of equation (5) only the first term in this expansion has been kept, therefore the applicability can be reduced to the condition of neglecting the second term. Since the function $\Delta$ is in the exponent, the condition is $\omega \boldsymbol{n} \ddot{\boldsymbol{\beta}}(\lambda / c)^{3} \ll 1$. The module of the particle velocity in magnetic field remains constant, $\boldsymbol{\beta}^{2}=$ const, thus $\frac{1}{2} \frac{d^{2}}{d t^{2}} \boldsymbol{\beta}^{2}=(\dot{\boldsymbol{\beta}})^{2}+\boldsymbol{\beta} \ddot{\boldsymbol{\beta}}=0$. Since emitted photons and the particle velocities are nearly parallel, $\boldsymbol{n} \approx \boldsymbol{\beta}$, the term $(\boldsymbol{n} \ddot{\boldsymbol{\beta}})$ can be estimated as $(\boldsymbol{n} \ddot{\boldsymbol{\beta}}) \approx(\boldsymbol{\beta} \ddot{\boldsymbol{\beta}})=-(\dot{\boldsymbol{\beta}})^{2}$. This gives the second condition of applicability of equation $(8): \omega(\dot{\boldsymbol{\beta}})^{2}(\lambda / c)^{3} \ll 1$. By expressing the acceleration $\dot{\boldsymbol{\beta}}$ through the magnetic field strength, the condition of applicability of equation (8) can be written in the form

$$
\omega \ll \frac{m^{2} c^{5} \gamma^{2}}{e^{2} B^{2} \lambda^{3}} .
$$

Note that for a homogeneous magnetic field $\lambda=\infty$, therefore the standard synchrotron spectrum cannot be derived in the framework of perturbation theory.

Equations (10) and (11) as conditions of applicability of the perturbation approach can be interpreted in the following way. If a charged particle travels in a region filled with magnetic field a path $\lambda$ which is shorter compared to the trajectory curvature $R$, then the particle is deflected by an angle $\delta \theta \simeq \lambda / R$. The first condition given by equation $(10)$ implies that $\delta \theta \ll 1 / \gamma$. Concerning the second condition given by equation (11), it is equivalent to the requirement that the segment of the trajectory of length of order $\sim \lambda$, can be treated as a straight line.

The characteristic frequency of the radiation in this regime, $\omega_{j}$, can be estimated from first principles. Namely, while the emission is formed during the time interval $\delta t_{\mathrm{rad}} \sim \lambda / c$, it is registered during $\delta t_{\mathrm{obs}}=$ $\delta t_{\text {rad }}(1-\boldsymbol{n} \boldsymbol{\beta}) \sim \delta t_{\text {rad }} / \gamma^{2}$. Thus the characteristic frequency is estimated as (see e.g. Landau \& Lifshit 1975)

$$
\omega_{j}=\frac{1}{\delta t_{\mathrm{obs}}}=\frac{c \gamma^{2}}{\lambda} .
$$

Note that this frequency $\omega_{j}$ is independent on the magnetic field strength $B$.

It is interesting to compare the characteristic frequencies, at which the bulk of radiation is produced, in highly turbulent and homogeneous magnetic fields corresponding to the jitter and synchrotron radiation regimes. The characteristic synchrotron frequency can be expressed through the nonrelativistic Larmor radius $R_{L}=m c^{2} / e B$ :

$$
\omega_{c}=\frac{3 c \gamma^{2}}{2 R_{L}}=\frac{3}{2} \frac{\lambda}{R_{L}} \omega_{j} .
$$

It is convenient to express equations (10) and (11) also through $R_{L}$ :

$$
\frac{\lambda}{R_{L}} \ll 1, \quad \omega \ll \omega_{j}\left(\frac{R_{L}}{\lambda}\right)^{2},
$$

When these conditions are satisfied, the ratio $\omega_{j} / \omega_{c} \sim$ $R_{L} / \lambda \gg 1$, i.e. the characteristic energy of photons emitted by charged particles in highly turbulent magnetic field may significantly exceed, by a factor of $R_{L} / \lambda$, the characteristic energy of synchrotron photons emitted by same particles in a regular magnetic field of same strength.

Finally, one should mention another constraint on applicability of equation (8) related to the plasma effects. The basic equation (2) describes emission in vacuum neglecting the impact of the surrounding plasma. If the radiating particle is located in plasma, the latter in the frequency range $\omega \gg \omega_{p}$ can be treated as a medium with dielectric permittivity $\epsilon(\omega)=1-\omega_{p}^{2} / \omega^{2}$, where

$$
\omega_{p}=\sqrt{\frac{4 \pi e^{2} n_{e}}{m_{e}}},
$$

is the plasma frequency $\left(n_{e}, m_{e}\right.$ and $e$ are the number density, mass and charge of electrons, respectively). At $\epsilon(\omega) \approx 1$, the term $(1-\boldsymbol{n} \boldsymbol{\beta}) \approx\left(\frac{1}{\gamma^{2}}+\theta^{2}\right) / 2$ in all above derived formulas should be replaced by the one corrected for the dielectric permittivity, $(1-\sqrt{\epsilon} \boldsymbol{n} \boldsymbol{\beta}) \approx\left(\frac{1}{\gamma^{2}}+\frac{\omega_{p}^{2}}{\omega^{2}}+\right.$ $\left.\theta^{2}\right) / 2$. Thus, the influence of the medium can be ignored for sufficiently high frequencies, $\omega \gg \omega_{p} \gamma$. Note that the particle Lorentz factor $\gamma$ and the plasma frequency enter the equation in the form of the combination $1 / \gamma^{2}+$ $\left(\omega_{p} / \omega\right)^{2}$. Therefore, the influence of the medium can be taken into account if in all above equations we replace the particle Lorentz factor $\gamma$ to

$$
\gamma^{*}(\omega)=\frac{\gamma}{\sqrt{1+\left(\omega_{p} \gamma / \omega\right)^{2}}} .
$$

(see e.g. Ginzburg \& Syrovatskii 1969; Fleishman 2006). However, as long as we are interested in high frequency range $\omega \gg \omega_{p} \gamma$, for the sake of simplicity we will ignore (unless otherwise is stated) the difference between $\gamma$ and $\gamma^{*}$.

\section{DEALING WITH CHAOTIC MAGNETIC FIELD}

The integrand of equation (8) contains the term

$$
\begin{gathered}
\left(\boldsymbol{a}_{+} \boldsymbol{a}_{-}\right)=\frac{e^{2}}{m^{2} \gamma^{2}}\left(\boldsymbol{\beta} \times \boldsymbol{B}_{+}\right)\left(\boldsymbol{\beta} \times \boldsymbol{B}_{-}\right) \\
=\frac{e^{2} \beta^{2}}{m^{2} \gamma^{2}}\left(\delta_{\rho \sigma}-\nu_{\rho} \nu_{\sigma}\right) B_{+\rho} B_{-\sigma},
\end{gathered}
$$

which should be averaged over different configurations of the magnetic field. Here $\boldsymbol{\nu}=\boldsymbol{\beta} /|\boldsymbol{\beta}|$ is unit velocity vector. The magnetic field values $\boldsymbol{B}_{+}$and $\boldsymbol{B}_{-}$corresponds to the points where the charged particle is located at time instants $(t \pm \tau / 2)$, i.e. $\boldsymbol{B}_{ \pm}=\boldsymbol{B}(\boldsymbol{r}(t) \pm \boldsymbol{\beta}(t) \tau / 2, t \pm \tau / 2)$. The statistical averaging of this expression will result in appearance of the correlation function:

$$
K_{\rho \sigma} \equiv\left\langle B_{\rho}\left(\boldsymbol{r}_{1}, t_{1}\right) B_{\sigma}\left(\boldsymbol{r}_{2}, t_{2}\right)\right\rangle,
$$


which is a second order tensor. Here, under the statistical averaging we suppose a general standard procedure; it could be a space-time homogenization or an integration over an ensemble of field configurations (see e.g. $§ 118$ of Landau \& Lifshitz 1980). Here we assume that the field is statistically homogeneous and stationary. This implies that the correlation function depends only on the difference of the coordinates $\left(\boldsymbol{r}_{1}-\boldsymbol{r}_{2}\right)$ and times $\left(t_{1}-t_{2}\right)$, i.e. $K_{\rho \sigma}=K_{\rho \sigma}\left(\boldsymbol{r}_{1}-\boldsymbol{r}_{2}, t_{1}-t_{2}\right)$. In this case, $\left\langle\boldsymbol{B}^{2}\right\rangle=$ $K_{\rho \rho}(0)=$ const

It is convenient to present the correlation function $K_{\rho \sigma}$ in the form of a Fourier integral:

$$
K_{\rho \sigma}(\boldsymbol{r}, t)=\int \tilde{K}_{\rho \sigma}(\boldsymbol{q}, \varkappa) e^{i(\boldsymbol{q} \boldsymbol{r}-\varkappa t)} \frac{d^{3} q}{(2 \pi)^{3}} \frac{d \varkappa}{2 \pi} .
$$

Since the magnetic field is divergence free $(\nabla \boldsymbol{B}=0)$, $K_{\rho \sigma}$ should satisfy the following conditions

$$
\partial K_{\rho \sigma} / \partial x_{\rho}=0, \quad \partial K_{\rho \sigma} / \partial x_{\sigma}=0,
$$

which for the Fourier transform $\tilde{K}_{\rho \sigma}$ take on form (the transversality condition):

$$
\tilde{K}_{\rho \sigma} q_{\rho}=0, \quad \tilde{K}_{\rho \sigma} q_{\sigma}=0 .
$$

While in Section 7 we will briefly discuss different tensor structures of the correlation function, here we consider the case of isotropic turbulence. This results in the following form of the correlation function (see e.g. Fleishman 2005):

$$
\tilde{K}_{\rho \sigma}(\boldsymbol{q}, \varkappa)=\frac{1}{2}\left(\delta_{\rho \sigma}-\frac{q_{\rho} q_{\sigma}}{q^{2}}\right) \Psi(|\boldsymbol{q}|, \varkappa)\left\langle\boldsymbol{B}^{2}\right\rangle .
$$

Here the constant factor $\left\langle\boldsymbol{B}^{2}\right\rangle$ is introduced which allows $\Psi$ to meet the normalization condition

$$
\int \Psi(q, \varkappa) \frac{d^{3} q}{(2 \pi)^{3}} \frac{d \varkappa}{2 \pi}=\frac{1}{4 \pi^{3}} \int_{-\infty}^{\infty} d \varkappa \int_{0}^{\infty} d q q^{2} \Psi(q, \varkappa)=1 .
$$

The tensor structure given by equation (22), obviously satisfies the transversality condition of equation (21).

The averaged values of $\left(\boldsymbol{a}_{+} \boldsymbol{a}_{-}\right)$can be expressed through the correlation function as

$$
\left\langle\boldsymbol{a}_{+} \boldsymbol{a}_{-}\right\rangle=\frac{e^{2}}{m^{2} \gamma^{2}}\left(\delta_{\rho \sigma}-\nu_{\rho} \nu_{\sigma}\right) K_{\rho \sigma}(c \boldsymbol{\beta} \tau, \tau) .
$$

Here we took into account that $\boldsymbol{r}_{+}-\boldsymbol{r}_{-}=c \boldsymbol{\beta} \tau$, and replaced in the numerator $\beta^{2}$ to 1 . From equations (19) and (22) we find

$$
\begin{gathered}
\int_{-\infty}^{\infty}\left\langle\left(\boldsymbol{a}_{+} \boldsymbol{a}_{-}\right)\right\rangle e^{i \tilde{\omega} \tau} d \tau=\frac{e^{2}}{2 m^{2} \gamma^{2}}\left\langle\boldsymbol{B}^{2}\right\rangle \\
\times \int\left(1+\frac{(\boldsymbol{\nu} \boldsymbol{q})^{2}}{q^{2}}\right) \Psi(q, \varkappa) e^{i(c \boldsymbol{q} \boldsymbol{\beta}-\varkappa+\tilde{\omega}) \tau} \frac{d^{3} q}{(2 \pi)^{3}} \frac{d \varkappa}{2 \pi} d \tau .
\end{gathered}
$$

After substitution of $\boldsymbol{\beta}$ by the velocity unit vector $\boldsymbol{\nu}$, and integration over $d \tau$ resulting in a $\delta$-function $2 \pi \delta(c \boldsymbol{q} \boldsymbol{\nu}-\varkappa+\tilde{\omega})$, the integral over $d \varkappa$ can be computed analytically:

$$
\int_{-\infty}^{\infty}\left\langle\boldsymbol{a}_{+} \boldsymbol{a}_{-}\right\rangle e^{i \tilde{\omega} \tau} d \tau=\frac{e^{2}}{2 m^{2} \gamma^{2}}\left\langle\boldsymbol{B}^{2}\right\rangle
$$

$$
\times \int\left(1+\frac{(\boldsymbol{\nu} \boldsymbol{q})^{2}}{q^{2}}\right) \Psi(q, \tilde{\omega}+c \boldsymbol{q} \boldsymbol{\nu}) \frac{d^{3} q}{(2 \pi)^{3}} .
$$

Thus, we arrive at the following expression for the energy and angular distribution of radiation per unit time

$$
\begin{gathered}
P_{\boldsymbol{n} \omega}(t)=\frac{e^{4}}{2 \pi^{2} m^{2} c^{3}}\left\langle\boldsymbol{B}^{2}\right\rangle \frac{\gamma^{2}\left(1+\gamma^{4} \theta^{4}\right)}{\left(1+\gamma^{2} \theta^{2}\right)^{4}} \\
\times \int\left(1+\frac{(\boldsymbol{\nu} \boldsymbol{q})^{2}}{q^{2}}\right) \Psi(q, \tilde{\omega}+c \boldsymbol{q} \boldsymbol{\nu}) \frac{d^{3} q}{(2 \pi)^{3}},
\end{gathered}
$$

where $\tilde{\omega}$ is determined by equation (9).

Let's consider now the case of steady turbulence, i.e. when the correlation function given by equation (18) is time-independent. Then the Fourier image of the correlation function contains a $\delta$-function, $\Psi(q, \varkappa)=$ $\Psi(q) 2 \pi \delta(\varkappa)$, and the normalization condition (23) becomes

$$
\int \Psi(q) \frac{d^{3} q}{(2 \pi)^{3}}=\frac{1}{2 \pi^{2}} \int_{0}^{\infty} \Psi(q) q^{2} d q=1 .
$$

Note that the function $\Psi$ determines the spectrum of the energy density of the stochastic magnetic field, since

$$
\frac{\left\langle\boldsymbol{B}^{2}\right\rangle}{8 \pi}=\frac{\left\langle\boldsymbol{B}^{2}\right\rangle}{16 \pi^{3}} \int_{0}^{\infty} \Psi(q) q^{2} d q .
$$

In the case of the stationary turbulence, a $\delta$-functional factor $2 \pi \delta(\tilde{\omega}+c \boldsymbol{\nu} \boldsymbol{q})$, appears in the integrand of equation (27). This makes the integration over $d \Omega_{\boldsymbol{q}}$ (note that $d^{3} q=q^{2} d q d \Omega_{\boldsymbol{q}}$ ) rather trivial:

$$
\begin{aligned}
& P_{\boldsymbol{n} \omega}(t)=\frac{e^{4}}{4 \pi^{3} m^{2} c^{4}}\left\langle\boldsymbol{B}^{2}\right\rangle \frac{\gamma^{2}\left(1+\gamma^{4} \theta^{4}\right)}{\left(1+\gamma^{2} \theta^{2}\right)^{4}} \\
& \times \int_{\tilde{\omega} / c}^{\infty}\left(1+\frac{\tilde{\omega}^{2}}{c^{2} q^{2}}\right) \Psi(q) q d q .
\end{aligned}
$$

Now we can conduct analytical integration over the emitting angles of radiation. The major contribution to the integral comes from range of small angles $\theta \lesssim 1 / \gamma$; the contribution from large angles, $\theta \gg 1 / \gamma$, is negligibly small. Thus, applying the standard approach for calculations of radiation of ultra-relativistic particles, one can adopt $d \Omega=2 \pi \theta d \theta$, and perform integration over $\theta$ from zero to infinity. It is also convenient to introduce a new integration variable $\zeta=\gamma^{2} \theta^{2}$ and change the order of integration over $\zeta$ and $q$. After performing a trivial integration over $\zeta$, we arrive at

$$
P_{\omega}(t)=\frac{e^{4}\left\langle\boldsymbol{B}^{2}\right\rangle}{6 \pi^{2} m^{2} c^{4}} \int_{\omega /\left(2 c \gamma^{2}\right)}^{\infty} u(\xi) \Psi(q) q d q,
$$

where $\xi=2 q c \gamma^{2} / \omega$, and

$$
u(\xi)=1+\frac{3}{\xi^{2}}-\frac{4}{\xi^{3}}-\frac{3 \ln \xi}{\xi^{2}} .
$$

In the range of integration over $d q$, the variable $\xi$ alters from 1 to $\infty$; while the function $u(\xi)$ increases 
monotonically from $u(1)=0$ to $u(\infty)=1$. Adopting $\xi$ as the integration variable, equation (31) can be presented in the form convenient for numerical computations:

$$
P_{\omega}(t)=\frac{e^{4}\left\langle\boldsymbol{B}^{2}\right\rangle}{6 \pi^{2} m^{2} c^{4}} \int_{1}^{\infty} u(\xi)\left(\frac{\omega \xi}{2 c \gamma^{2}}\right)^{2} \Psi\left(\frac{\omega \xi}{2 c \gamma^{2}}\right) \frac{d \xi}{\xi}
$$

Equation (31) is an integral function which depends on the turbulence spectrum. However, it obeys some general properties not affected by the turbulence. In particular, from equation (31) follows that independent of $\Psi(q)$, the radiation spectrum $P_{\omega}(t)$ is a monotonically decreasing function of $\omega$. This feature becomes obvious after the differentiation over $\omega$ :

$$
\frac{\partial P_{\omega}}{\partial \omega} \sim \int_{\omega /\left(2 c \gamma^{2}\right)}^{\infty} \frac{\partial \xi}{\partial \omega} \frac{d u(\xi)}{d \xi} \Psi(q) q d q
$$

Here it is taken into account that the contribution to the derivative from the lower integration limit is null (given that $u(1)=0)$. Since $d u(\xi) / d \xi>0, \Psi(q) \geq 0$ and $\partial \xi / \partial \omega<0$, the integrand is negative, and the integration results in $\partial P_{\omega} / \partial \omega<0$. Thus, this function achieves its maximum value at $\omega=0$, i.e.,

$$
P_{\omega}(t) \leq P_{0}=\frac{e^{4}\left\langle\boldsymbol{B}^{2}\right\rangle}{6 \pi^{2} m^{2} c^{4}} \int_{0}^{\infty} \Psi(q) q d q .
$$

Of course, this estimate is meaningful only if the integral in the right side of equation converges.

Note that the photon energy and the particle Lorentz factor enter to equation (31) only in the form of ratio $\omega / \gamma^{2}$. Thus, the spectrum $P_{\omega}$ is, in fact, a function of one argument $\omega / \omega_{j}$ (for intermediate calculations we drop, just for simplicity, the argument $t)$ :

$$
P_{\omega} \equiv \tilde{P}\left(\frac{\omega}{\omega_{j}}\right)=\tilde{P}\left(\frac{\lambda \omega}{c \gamma^{2}}\right)
$$

Here $\tilde{P}\left(\omega / \omega_{j}\right)$ is a monotonically decreasing function. In case of absence of other characteristic frequencies in the physical setup except for $\omega_{j}$, in the range $\omega \ll \omega_{j}$ function $\tilde{P}$ is nearly constant, $\tilde{P} \approx P_{0}$. However, at very small frequencies the surrounding plasma may significantly change the behavior of $P_{\omega}$. The substitution of $\gamma$ by $\gamma_{*}$ (in accordance with equation (16)) leads to equation (31) in which $\xi$ should be replaced by $\xi_{*}=2 q c \gamma_{*}^{2} / \omega$.

The derivative

$$
\frac{\partial \xi_{*}}{\partial \omega}=-2 q c \gamma^{2} \frac{\omega^{2}-\gamma^{2} \omega_{p}^{2}}{\left(\omega^{2}+\gamma^{2} \omega_{p}^{2}\right)^{2}},
$$

has a positive sign at $\omega<\gamma \omega_{p}$, and becomes negative when $\omega>\gamma \omega_{p}$. Therefore, independent of the choice of the spectrum of turbulence $\Psi(q)$, the emission intensity increases with frequency in the range of $\omega<\gamma \omega_{p}$, and decreases when $\omega>\gamma \omega_{p}$, while the maximum is reached at $\omega=\gamma \omega_{p}$. Then, instead of equation (36), we have

$$
P_{\omega}=\tilde{P}\left[\frac{\lambda}{c \gamma^{2}}\left(\omega+\frac{\gamma^{2} \omega_{P}^{2}}{\omega}\right)\right]
$$

The argument of this function has a minimum at $\omega=$ $\gamma \omega_{p}$, and consequently the function achieves its maximum at this frequency. However, we should note that in the case of convergence of the integral in equation (32), this maximum would be practically invisible. To demonstrate the behavior of $P_{\omega}$ at small frequencies, in figure 2 we show calculations for three different turbulence spectra $\Psi$ presented in the following specific form

$$
\Psi(q)=\frac{A_{\alpha_{1}}}{q^{2+\alpha_{1}}\left(1+\lambda^{2} q^{2}\right)^{1-\alpha_{1} / 2}} .
$$

Here, according to equation (28), the normalization constant

$$
A_{\alpha_{1}}=4 \pi^{3 / 2} \lambda^{1-\alpha_{1}} \frac{\Gamma\left(1-\alpha_{1} / 2\right)}{\Gamma\left(\left(1-\alpha_{1}\right) / 2\right)},
$$

where $\Gamma(z)$ is the gamma-function. The results of calculations in figure 2 correspond to three different values of $\alpha_{1}: \alpha_{1}=-1,0$ and $1 / 2$. It can be seen that while for $\alpha_{1}=0$ or $1 / 2$ the integral in equation (32) diverges and the maximum of $P_{\omega}$ is clearly seen at $\gamma \omega_{p}$, for the value of $\alpha_{1}=-1$ the emission intensity is characterized by a broad plateau without any distinct maximum.

To explore the emission spectra in the frequency range $\gamma \omega_{p} \ll \omega \ll \omega_{j}$ and $\omega \gg \omega_{p}$, let's assume that the turbulence spectrum has a broken power-law form:

$$
\Psi(q)= \begin{cases}\lambda^{3}\left(\frac{q_{1}}{q}\right)^{2+\alpha_{1}}, & q \ll \frac{1}{\lambda}, \\ \lambda^{3}\left(\frac{q_{2}}{q}\right)^{2+\alpha_{2}}, & q \gg \frac{1}{\lambda},\end{cases}
$$

where $q_{1}$ and $q_{2}$ are constants of the order of $1 / \lambda$, and the factor $\lambda^{3}$ is introduced for the reason of dimension consistency. The condition for the convergence of the integral in equation (28) on the lower and upper limits implies

$$
\alpha_{1}<1, \quad \alpha_{2}>1
$$

Depending on the value of $\alpha_{1}$ there are two different cases related to the convergence of the integral in equation (32). If the integral is converging at the lower limit (i.e., $\alpha_{1}<0$ ), we have the case discussed above. Let's consider now the range of $0<\alpha_{1}<1$. Then, for the frequency interval $\gamma \omega_{P} \ll \omega \ll \omega_{j}$ we have

$$
P_{\omega}=\frac{e^{4} \lambda^{3} q_{1}^{2}\left\langle\boldsymbol{B}^{2}\right\rangle}{2 \pi^{2} m^{2} c^{4}}\left[\left(\frac{2 c \gamma^{2} q_{1}}{\omega}\right)^{\alpha_{1}}-1\right] \frac{C_{1}}{\alpha_{1}},
$$

where

$$
C_{1}=\frac{4+3 \alpha_{1}+\alpha_{1}^{2}}{\left(3+\alpha_{1}\right)\left(2+\alpha_{1}\right)^{2}} .
$$

At lower frequencies, $\omega_{P} \ll \omega \ll \gamma \omega_{P}$,

$$
P_{\omega}=\frac{e^{4} \lambda^{3} q_{1}^{2}\left\langle\boldsymbol{B}^{2}\right\rangle}{2 \pi^{2} m^{2} c^{4}}\left[\left(\frac{2 c \omega q_{1}}{\omega_{P}^{2}}\right)^{\alpha_{1}}-1\right] \frac{C_{1}}{\alpha_{1}} .
$$

We note that these equations (43, 45) allow a smooth passage to the limit $\alpha_{1} \rightarrow 0$.

In the range of large frequencies, $\omega \gg \omega_{j}$, the radiation spectrum behaves as a power-law $\omega^{-\alpha_{2}}$, i.e. mimics the turbulence spectrum (TF87),

$$
P_{\omega}=\frac{e^{4} \lambda^{3} q_{2}^{2}\left\langle\boldsymbol{B}^{2}\right\rangle}{6 \pi^{2} m^{2} c^{4}}\left(\frac{2 c \gamma^{2} q_{2}}{\omega}\right)^{\alpha_{2}} C_{2},
$$




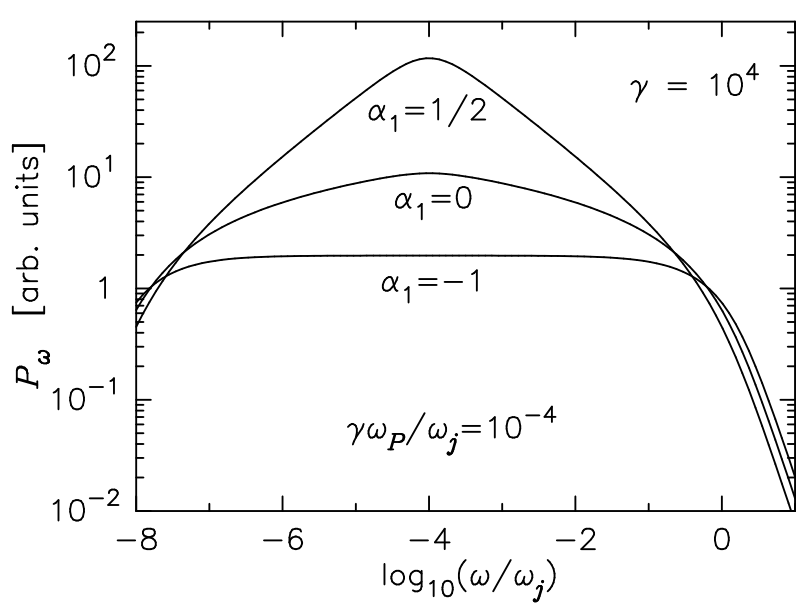

FIG. 2.- Spectral power calculated for the turbulence spectrum $\Psi$ given in the form of equation (39) for three different values of power-law index $\alpha_{1}$.

where

$$
C_{2}=\frac{1}{\alpha_{2}}+\frac{3}{1+\alpha_{2}}-\frac{3}{\left(2+\alpha_{2}\right)^{2}}-\frac{4}{3+\alpha_{2}} .
$$

The energy lose rate of a charged particle due to radiation in the magnetic field is given by the classical formula (see e.g. Landau \& Lifshitz 1975)

$$
I=\frac{2 e^{4} \gamma^{2}}{3 m^{2} c^{3}}(\boldsymbol{\beta} \times \boldsymbol{B})^{2}=\frac{2 e^{4} \beta^{2} \gamma^{2}}{3 m^{2} c^{3}} B^{2} \sin ^{2} \chi,
$$

where $\chi$ is angle between the particle velocity and direction of the magnetic field. By averaging $I$, first over the directions, then over the strength of the magnetic field, and taking into account that $\left\langle\sin ^{2} \chi\right\rangle=\frac{2}{3}$, one finds

$$
I=\frac{4 e^{4} \gamma^{2}}{9 m^{2} c^{3}}\left\langle B^{2}\right\rangle
$$

(in the numerator, $\beta^{2}=1$ is substituted). By definition, the same result can be obtained by direct integration of equation (31) or equation (33) over the emitted photon frequencies: $\int_{0}^{\infty} P_{\omega} d \omega=I$. Nevertheless, it is worth to perform we such computations; they can serve as a good test for the consistency of the results.

From equation (33) we find

$$
\int_{0}^{\infty} P_{\omega} d \omega=\frac{e^{4}\left\langle\boldsymbol{B}^{2}\right\rangle}{6 \pi^{2} m^{2} c^{4}} \int_{1}^{\infty} \frac{d \xi}{\xi} u(\xi) \int_{0}^{\infty} d \omega\left(\frac{\omega \xi}{2 c \gamma^{2}}\right)^{2} \Psi\left(\frac{\omega \xi}{2 c \gamma^{2}}\right) .
$$

After the substitution of the new variable $\omega=q \times$ $\left(2 c \gamma^{2}\right) / \xi$, the integration over $\omega$ leads to equation (28), and then we obtain

$$
\int_{0}^{\infty} P_{\omega} d \omega=\frac{2 e^{4} \gamma^{2}\left\langle\boldsymbol{B}^{2}\right\rangle}{3 m^{2} c^{3}} \int_{1}^{\infty} \frac{d \xi}{\xi^{2}} u(\xi) .
$$

The remaining integral is equal to $2 / 3$, so the direct integration of the emission spectrum leads to equation (49). This interesting result, when the integration of the $a p$ proximate equation (33) gives precise expression for energy losses, has a quite natural explanation; while equation (33) contains the first (quadratic) term in the ex- pansion of the spectrum over the magnetic field strength $B$, the precise expression for the energy losses given by equation (49) is proportional to the second power of $B$. Also we note that the energy losses are independent of the spectrum of turbulence $\Psi$.

In a similar way one can find the angular distribution of the emission after integration over the frequencies; therefore we simply write down the final result:

$$
d I_{\boldsymbol{n}}=\frac{4 e^{4} \gamma^{4}\left\langle\boldsymbol{B}^{2}\right\rangle}{3 \pi m^{2} c^{3}} \frac{1+\gamma^{4} \theta^{4}}{\left(1+\gamma^{2} \theta^{2}\right)^{5}} d \Omega .
$$

Here, $d I_{n}$ is the energy emitted into the solid angle $d \Omega$ per time unit. This angular distribution also does not depend on the turbulence spectrum $\Psi$.

\section{LARGE SCALE TURBULENCE}

In the case of a large scale magnetic field turbulence, $\lambda \gtrsim R_{L}$, the conditions imposed by equation (14) are violated, therefore the results of the previous section are not anymore valid. On the other hand, the radiation spectrum formed in the regime $\lambda \gg R_{L}$ can be derived analytically. In this case the particle deflection angle exceeds $1 / \gamma$, and the radiation spectrum, $P_{\omega}$, is determined by the instant curvature of trajectory (or the instant value of the magnetic field). Thus the result should be similar to the spectrum of synchrotron radiation in the homogeneous magnetic field (Schwinger 1949: Ginzburg \& Sycovatskii 1969; Landau \& Lifshitz 1975). If the charged particle moves perpendicularly to the magnetic field, the emission spectrum is determined as

$$
P_{\omega}(t)=\frac{\sqrt{3} e^{2}}{2 \pi R_{L}} F(x),
$$

where

$$
F(x)=x \int_{x}^{\infty} K_{5 / 3}(u) d u .
$$

Here $K_{5 / 3}(u)$ is the modified Bessel function, $x=\omega / \omega_{c}$ and $\omega_{c}$ is determined by equation (13). If the charged particle moves at an angle $\chi$ to the magnetic field, in equation (53) $B$ should be substituted by the perpendicular component of the field, $B_{\perp} \equiv B \sin \chi$ Ginzburg \& Syrovatskii 1969).

If the magnetic field is turbulent, then the spectrum $P_{\omega}(t)$ should be averaged over directions of the field, i.e., integrated over the pitch angle $\chi$. This results in the following expression (Crusius \& Schlickeisen 1986)

$$
P_{\omega}(t)=\frac{\sqrt{3} e^{2}}{2 \pi R_{L}} G(x),
$$

where

$$
G(x)=\frac{\pi x}{2}\left(W_{0, \frac{4}{3}}(x) W_{0, \frac{1}{3}}(x)-W_{\frac{1}{2}, \frac{5}{6}}(x) W_{-\frac{1}{2}, \frac{5}{6}}(x)\right) .
$$

Here $W_{\mu, \alpha}(x)$ is the Whittaker function.

The function $G(x)$ can be presented in a more convenient form:

$$
G(x)=\frac{x}{20}\left[\left(8+3 x^{2}\right)\left(\kappa_{1 / 3}\right)^{2}+x \kappa_{2 / 3}\left(2 \kappa_{1 / 3}-3 x \kappa_{2 / 3}\right)\right],
$$


via familiar Bessel functions $\kappa_{1 / 3}=K_{1 / 3}(x / 2), \kappa_{2 / 3}=$ $K_{2 / 3}(x / 2)$ (Aharonian et al. 2010). $G(x)$ has a simple asymptotic behavior both at ow and high frequencies:

$$
G(x) \approx \begin{cases}\frac{2^{1 / 3}}{5}(\Gamma(1 / 3))^{2} x^{1 / 3}, & x \ll 1, \\ \frac{\pi}{2} e^{-x}, & x \gg 1 .\end{cases}
$$

Although differences between the spectra of synchrotron radiation in homogeneous and (large scale) chaotic fields, i.e. between functions $F(x)$ and $G(x)$, are not dramatic, yet they not too small to be neglected in calculations (Aharonian et al. 2010). In particular, these functions achieve their maximums, $\max (F)=0.918$ and $\max (G)=0.713$, at different points, $x=0.286$ and $x=0.229$, respectively. Obviously, similar differences we should expect for the spectral energy distributions described by the functions $x F(x)$ and $x G(x)$. Namely, $\max (x F)=0.693$ is achieved at $x=1.33$, and $\max (x G)=0.444$ is achieved at $x=1.15$.

Finally, we note that function $G(x)$ can be approximated by a simple analytical expression,

$$
G(x)=\frac{1.808 x^{1 / 3}}{\sqrt{1+3.4 x^{2 / 3}}} \frac{1+2.21 x^{2 / 3}+0.347 x^{4 / 3}}{1+1.353 x^{2 / 3}+0.217 x^{4 / 3}} e^{-x},
$$

which provides better than $0.2 \%$ accuracy Aharonian et al. 2010). Thus, this approximation can be safely used, instead of the precise equation (57), in detailed calculations of radiation in environments with large scale turbulent magnetic field.

When deriving Eq.(55) we assumed that the magnetic field $\boldsymbol{B}$ is oriented chaotically, but its absolute value, $|\boldsymbol{B}|$, is fixed. However, in a turbulent medium the spatial variation of the field strength could be quite significant; therefore we have to average the results also over the absolute value of the field (see, e.g., Bykov et al. 2012). Let's introduce the distribution function

$$
w(B) d B=h_{n}(b) d B / B_{0},
$$

where $B_{0} \equiv \sqrt{\left\langle\boldsymbol{B}^{2}\right\rangle}, b=B / B_{0}$. By definition, $w(B) d B$ is the probability of the strength of the magnetic field being in the interval $(B, B+d B)$. We will consider three different distributions with the function $h_{n}(b)$ presented in the following forms:

$$
\begin{gathered}
h_{0}(b)=\delta(b-1), \\
h_{1}(b)=\frac{3 \sqrt{6}}{\sqrt{\pi}} b^{2} e^{-3 b^{2} / 2}, \\
h_{2}(b)=\frac{32 b^{2}}{\pi\left(1+b^{2}\right)^{4}},
\end{gathered}
$$

For all distributions

$$
\int_{0}^{\infty} h_{n}(b) d b=\int_{0}^{\infty} b^{2} h_{n}(b) d b=1 .
$$

For the variance of these distributions, $D=\left\langle b^{2}\right\rangle-\langle b\rangle^{2}=$ $1-\langle b\rangle^{2}$, we have

$$
D_{0}=0, \quad D_{1}=0.15, \quad D_{2}=0.28 .
$$

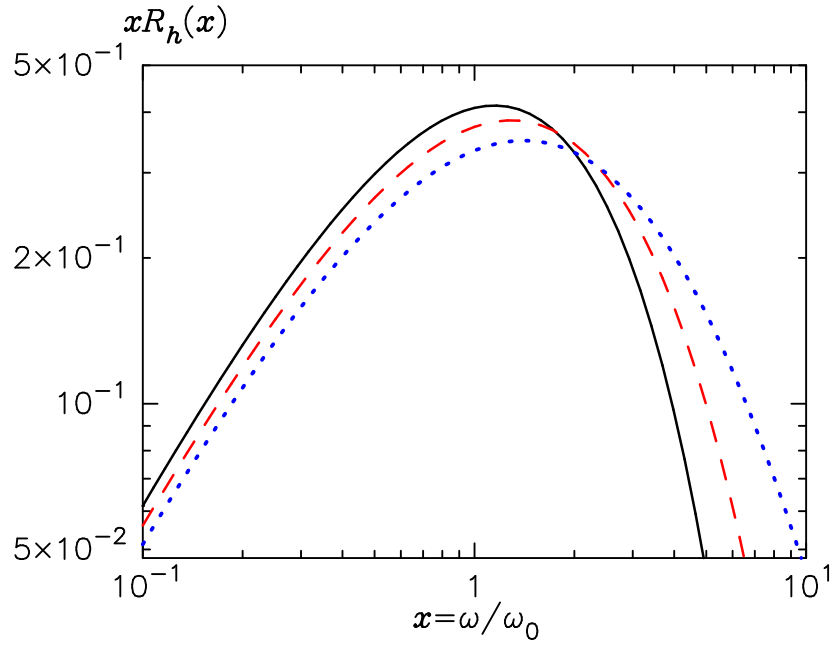

FIG. 3. - Spectral energy distribution of synchrotron radiation corresponding to distributions of the magnetic field $h_{0}$ (solid curve), $h_{1}$ (dashed curve), $h_{2}$ (dotted curve).

The energy lose rate of particles given by Eq.(49) depends only on $\left\langle\boldsymbol{B}^{2}\right\rangle$, therefore it is convenient to compare the averaged spectra for the same value of $\left\langle\boldsymbol{B}^{2}\right\rangle$ :

$$
\left\langle P_{\omega}\right\rangle \equiv \int_{0}^{\infty} P_{\omega} w(B) d B=I R_{h}(x) / \omega_{0},
$$

where $\omega_{0}=3 e B_{0} \gamma^{2} /(2 m c), x=\omega / \omega_{0}, \int_{0}^{\infty} R_{h}(x) d x=$ 1.

In Fig 3 we show the SED of synchrotron radiation, $x R_{h}(x)$, calculated for magnetic field distributions given by Eqs.(61),(62) and (63).

One can see that the spectrum of synchrotron radiation is somewhat shifted, depending on the distribution of the magnetic field, towards higher energies, and, more importantly, it is significantly broadened compared to the radiation spectrum relevant to the $\delta$-functional distribution of magnetic field $h_{0}$. One can show (see Appendix A that if $w(B)$ is characterized by a power-lay asymptotic dependence: $w(B) \propto B^{-\sigma}$ for $B \rightarrow \infty$, then the spectrum of synchrotron radiation also has a powerlaw asymptotic, namely $\langle P\rangle \propto \omega^{-\sigma+2}$ for $\omega \gg \omega_{0}$. Note that the power-law index should exceed $\sigma>3$ in order to provide convergence of $\left\langle\boldsymbol{B}^{2}\right\rangle$.

It is important to emphasize the broadening and the shift of the spectrum of synchrotron radiation in a largescale turbulent field has a quite different origin and should not be confused with the effects related to the jitter regime of radiation in a small-scale turbulent field. In this regard we should note that a broadening of synchrotron radiation has been "observed" in the numerical simulations of Teraki \& Takahara (2011). However, most likely the authors misinterpreted the obtained spectral feature and refereed it to an intermediate regime between the synchrotron and jitter regimes of radiation. However, we believe that this component of radiation revealed in their simulations, has a standard synchrotron origin, but simply broadened because of the distribution of the strength of the magnetic field. 


\section{ENERGY SPECTRA OF RADIATION IN THE JITTER AND SYNCHROTRON REGIMES}

In this section we compare spectra of synchrotron and jitter radiation, produced in two different large- and small- scale turbulent magnetic fields but with the same value $\left\langle\boldsymbol{B}^{2}\right\rangle$, thus the total radiation power given by equation (49) is the same for radiation in both regimes. For comparison, it is convenient to introduce the normalized the emission intensity:

$$
R(x) d x=P_{\omega} d \omega / I, \quad x=\omega / \omega_{c} .
$$

Obviously, the following condition is held: $\int_{0}^{\infty} R(x) d x=$ 1.

For calculations we have to select a assume a spectrum of turbulence and a distribution of the magnetic field strengths. For the sake of simplicity, below we consider the case of chaotic synchrotron emission, i.e., we adopt a field distribution corresponding to equation (61). Then the function $R$ depends only on the magnetic field,

$$
R(x)=\frac{27 \sqrt{3}}{16 \pi} G(x) .
$$

We note however that $R$ as a function of argument $x$ does not depend on $\left\langle\mathbf{B}^{2}\right\rangle$. In this section, for calculations of jitter radiation we consider the turbulence spectrum as an one-parameter family of functions:

$$
\Psi(q)=\frac{\lambda^{3} A_{\alpha}}{\left(1+\lambda^{2} q^{2}\right)^{1+\alpha / 2}} .
$$

The normalization constant, $A_{\alpha}$, is obtained from equation (28):

$$
A_{\alpha}=\frac{8 \pi^{3 / 2} \Gamma(1+\alpha / 2)}{\Gamma((\alpha-1) / 2)} .
$$

The spectrum presented in the form of equation (69) is characterized by a power-law dependence for $q \gg \lambda^{-1}$. Although the spectra of turbulence, which can be generated in astrophysical environments, remains an open question, usually it is approximated as a power-law. This assumption is justified by a few fundamental considerations. In particular, the power-law spectra of turbulence with spectral indices of $5 / 3$ and $3 / 2$ appear in the hydrodynamical (Kolmogorov 1941) and magnetohydrodynamical (Iroshnikov 1963; Kraichnan 1965) turbulent media.

Note that the asymptotic form of equation (69) is consistent with equation (41) for $\alpha_{1}=-2, \alpha_{2}=\alpha$. In figure 1 we show the normalized spectral energy distributions of the synchrotron and jitter radiation, $x R(x)$ produced by particles of fixed energy $\gamma m c^{2}$. The spectra are plotted as a function of $x=\epsilon / \epsilon_{c}=\hbar \omega / \hbar \omega_{c}$, for three different indices characterizing the turbulence, $\alpha=2,5 / 3,3 / 2$.

For a rather broad range of variation of the index $\alpha$, from $3 / 2$ to 3 , the presentation of the turbulence spectrum in the form of equation (69) allows simple analytical approximations for the radiation power

$$
P_{\omega} d \omega=I f\left(x_{j}\right) d \omega / \omega_{j},
$$

where $x_{j}=\omega / \omega_{j}$, and

$$
f\left(x_{j}\right)=C_{\alpha}\left(1+0.22 x_{j}+0.43 x_{j}^{2}\right)^{-\alpha / 2} .
$$

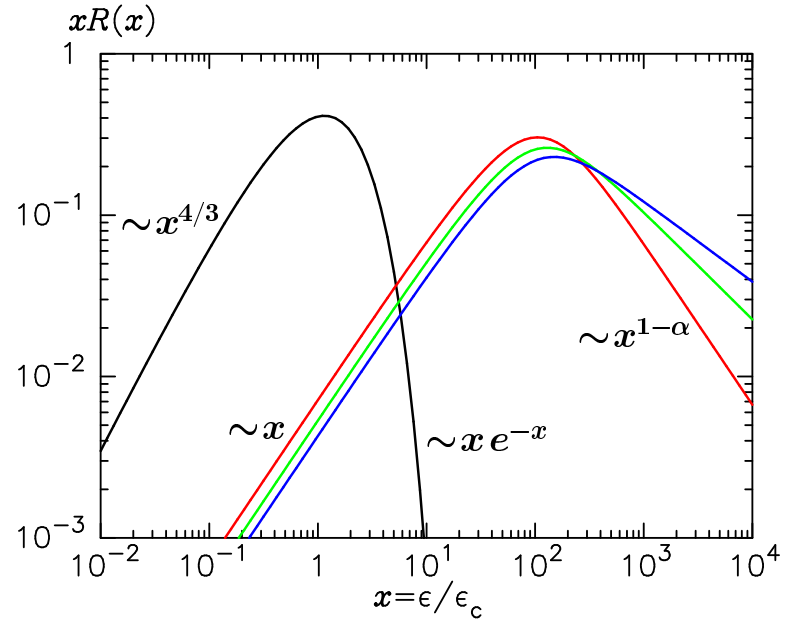

FIG. 4.- SED $(x R(x)$, see equation (67)) of radiation of monoenergetic particles in turbulent magnetic field in the synchrotron and jitter regimes: synchrotron (black line) and jitter (red, green and blue solid lines). The spectrum of turbulence was taken in the form of equation (69). The ratio of the correlation length to nonrelativistic Larmor radius $\left(R_{L}=m c^{2} / e B\right)$ was adopted to be $\lambda / R_{L}=10^{-2}$. Red, green and blue lines correspond to the indices $\alpha=2,5 / 3$ and $3 / 2$, respectively.

The coefficient $C_{\alpha}$ is determined from the normalization $\int_{0}^{\infty} f\left(x_{j}\right) d x_{j}=1$. The comparison with the exact numerical calculations shows that the precision of this approximation is better than $7 \%$.

Figure 1 demonstrates the basic spectral features of the jitter radiation. The SED peaks at energy which compared to the maximum of the synchrotron radiation at $\epsilon=1.155 \epsilon_{c}$ is shifted by the factor of $2 / 3 R_{L} / \lambda$. Below the maximum $x R(x) \propto x$, i.e. the SED increases with energy slower than the SED of the synchrotron radiation, $x R(x) \propto x^{4 / 3}$. Moreover, while the synchrotron spectrum has a quite sharp (exponential) cutoff beyond $x \sim 1$, the SED of jitter radiation after the break at $x \sim R_{L} / \lambda$ continues as a power-law, $x R(x) \propto x^{1-\alpha}$ up to $x \sim\left(R_{L} / \lambda\right)^{3}$.

In astrophysical environments, acceleration of particles typically leads to broad energy distributions. Below we compare the synchrotron and jitter radiations for different distributions of accelerated particles $N(\gamma)$ :

$$
P(\omega)=\int_{0}^{\infty} P_{\omega} N(\gamma) d \gamma .
$$

Here we assume that energy distribution of all particles occupies certain energy interval $\left(\gamma_{\min }, \gamma_{\max }\right)$. Outside this interval, the function $N$ is null 3 . For the jitter radiation, using equation (33) and introducing a new dimensionless function $\Psi_{1}(\lambda q)=\Psi(q) / \lambda^{3}$, as well as substituting the integration variable $\gamma$ by $\eta=\lambda \omega \xi /\left(2 c \gamma^{2}\right)$,

\footnotetext{
${ }^{3}$ We would like to indicate to the non-physical lower limit in the integral in equation (73). However, this convenient for integration representation is correct as long as the function $N$ is taken zero outside the physically meaningful region
} 
we obtain

$$
\begin{gathered}
P(\omega)=\frac{e^{4} \lambda\left\langle\boldsymbol{B}^{2}\right\rangle}{12 \pi^{2} m^{2} c^{4}} \int_{1}^{\infty} d \xi u(\xi) \\
\times \int_{0}^{\infty} d \eta \sqrt{\frac{\lambda \omega \eta}{2 c \xi}} \Psi_{1}(\eta) N\left(\sqrt{\frac{\lambda \omega \xi}{2 c \eta}}\right) .
\end{gathered}
$$

Let's assume now that the relativistic charged particles have a power-law distribution, $N(\gamma)=N_{0} \gamma^{-\mu}$. It can be shown that for the range of the power-law index, $1<\mu<$ $2 \alpha+1$, the main contribution to equation $(73)$ is provided by particles of energy $\gamma \sim(\lambda \omega / c)^{1 / 2}$. Therefore, for the energy interval $\omega \gg c / \lambda$, equation (74) can be integrated over $d \eta$ in the limits from 0 to $\infty$ :

$$
\begin{gathered}
P(\omega)=\frac{e^{4} \lambda\left\langle\boldsymbol{B}^{2}\right\rangle}{12 \pi^{2} m^{2} c^{4}}\left(\frac{2 c}{\lambda \omega}\right)^{(\mu-1) / 2} \\
\times \int_{1}^{\infty} d \xi u(\xi) \xi^{-(\mu+1) / 2} \int_{0}^{\infty} d \eta \Psi_{1}(\eta) \eta^{(\mu+1) / 2} .
\end{gathered}
$$

The power-law dependence of the spectra $(P(\omega) \sim$ $\left.\omega^{-(\mu-1) / 2}\right)$ is explained by the same reason as in the case of the synchrotron radiation: $\omega$ and $\gamma$ enter into $P_{\omega}$ in a combined form $\omega / \gamma^{2}$ (for discussion of the case of synchrotron radiation see Rybicki \& Lightman 1979). Thus, for a power-law particle distribution the synchrotron and jitter mechanisms lead to the same type of energy spectra, therefore the ratio of the emission intensities due to these two processes,

$$
r \equiv \frac{P_{\mathrm{jitt}}(\omega)}{P_{\mathrm{synchr}}(\omega)}=C(\mu, \alpha)\left(\frac{R_{L}}{\lambda}\right)^{(\mu-3) / 2},
$$

does not depend on photon energy $\omega$. Interestingly, the index of $\mu=3$ appears to be special, in the sense that independently of the turbulence spectrum, the ratio $r=$ 1. This, in particular, can be seen in figure 5 at low energies. Note that although the energy losses due to the synchrotron and jitter mechanisms in the large and small turbulent fields are equal (for the same mean magnetic field), formally for $\mu>3$ larger energy is radiated out due to the jitter mechanism $(r>1)$, and vise versa, $r<$ 1 for $\mu<3$. This apparent inconsistency is related to the assumption of pure power-law particle distribution. However, for a realistic distribution of particles with a high energy cutoff, the spectral shape of the synchrotron and jitter radiations differ significantly. In particular, for power-law distributions with an exponential cutoff, given in a rather general form

$$
N \sim \gamma^{-\mu} \exp \left(-\left(\gamma / \gamma_{\text {cut }}\right)^{\beta}\right),
$$

in the high energy limit, the shapes of the synchrotron and jitter radiations spectra differ significantly (see figure 5). While the synchrotron component beyond the maximum decreases exponentially (Lefa et al. 2012, also see Fritz 1989; Zirakashvili \& Aharonian 2007):

$$
P(\omega) \propto \exp \left[-\frac{\beta+2}{2}\left(\frac{2 \omega}{\omega_{\text {cut }}}\right)^{\beta /(\beta+2)}\right],
$$

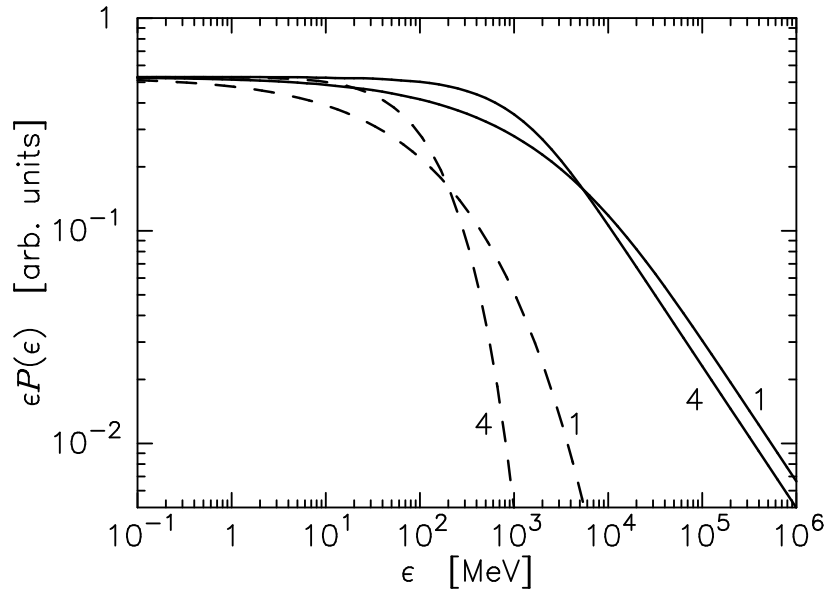

FIG. 5.- SED of synchrotron (dashed lines) and jitter (solid lines) radiation calculated for electron distribution: $\gamma^{-3} \exp \left[-\left(\gamma / \gamma_{\text {cut }}\right)^{\beta}\right]$. Calculations are performed for two values of $\beta$ : $\beta=1$ and $\beta=4$ (the number labels indicate the used values for different lines ). The cutoff energy is set $\gamma_{\text {cut }}=10^{8}$, and the computations are performed for $B=1 \mathrm{G}$. The jitter radiation is computed for turbulence spectrum in the form of equation (39) with $\alpha=5 / 3$ and the ratio of the field correlation length to the nonrelativistic Larmor radius $\left(R_{L}=m c^{2} / e B\right)$ is $\lambda / R_{L}=0.1$.

with

$$
\omega_{\text {cut }}=\frac{3 e B}{2 m c} \gamma_{\text {cut }}^{2},
$$

the jitter emission spectrum beyond the break around $\omega_{\text {cut }}\left(R_{L} / \lambda\right)$, has a long power-law tail, $P(\omega) \propto \omega^{-\alpha}$, independently of the shape of the particle distribution in the cutoff region (i.e. the value of $\beta$ ). In this regard, this is a unique feature of the jitter radiation which provides direct and model-independent information about the spectrum of turbulence. As long as the condition of small-scale turbulence is satisfied $\left(\lambda<R_{L}\right)$, we should expect radiation with characteristic a broken power-law type spectrum. While the photon index at low energies is directly related to the spectral index of relativistic particles, $\Gamma=(\mu+1) / 2$, or in the case of a low energy cutoff or very hard particle spectrum below the cutoff energy (e.g. in the case of Maxwellian type distribution - see figure 6) , $\Gamma=1$, the spectrum after the break depend only on the spectrum of turbulence. In an environment with large scale turbulence, the picture is just opposite. The radiation proceeds in the synchrotron regime and therefore is not sensitive to the details of the turbulence. On the other hand, the synchrotron radiation carries information about the overall spectrum of parent particles, including the most important (from the point of view of the acceleration theory) cutoff region.

In Fig. 6 we show a comparison of the synchrotron and jitter radiation for the case of Maxwell distribution of emitting particles.

\section{ANISOTROPIC TURBULENCE}

If the distribution of the charged particles is isotropic, the analytical solutions derived in the previous sections can be generalized to the case of the correlation tensor with an arbitrary angular structure. Indeed, similarly to 


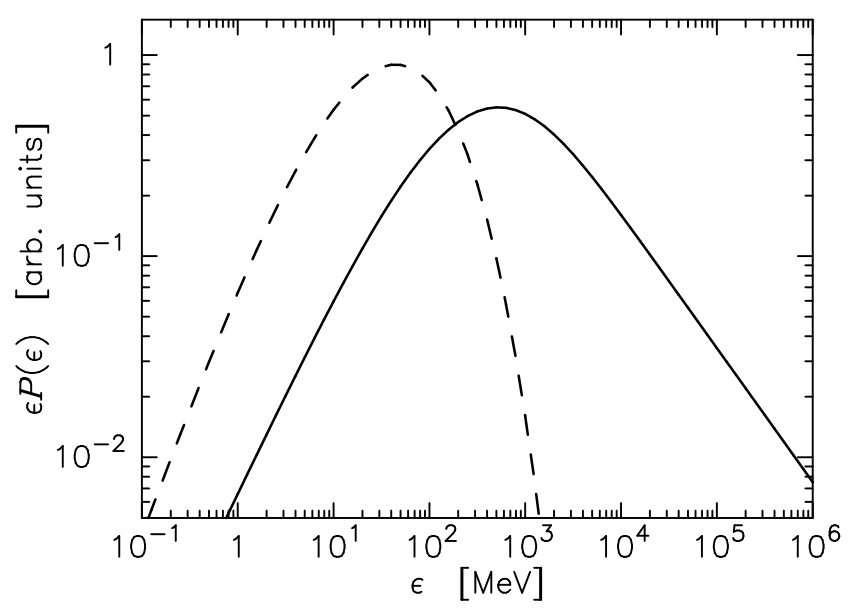

Fig. 6.- The same as in figure 月, but for Maxwelliantype distribution of charged particles: $\gamma^{2} \exp \left(-\gamma / \gamma_{\text {cut }}\right)$ with $\gamma_{\text {cut }}=10^{7}$.

equation (27), the radiation power can be expressed as

$$
\begin{aligned}
& P_{\boldsymbol{n} \omega}(t)=\frac{e^{4}}{\pi^{2} m^{2} c^{3}} \frac{\gamma^{2}\left(1+\gamma^{4} \theta^{4}\right)}{\left(1+\gamma^{2} \theta^{2}\right)^{4}}\left(\delta_{\rho \sigma}-\nu_{\rho} \nu_{\sigma}\right) \\
& \times \int \tilde{K}_{\rho \sigma}(\boldsymbol{q}, \varkappa) 2 \pi \delta(\tilde{\omega}+c \boldsymbol{q} \boldsymbol{\nu}-\varkappa) \frac{d^{3} q}{(2 \pi)^{3}} \frac{d \varkappa}{2 \pi} .
\end{aligned}
$$

To obtain the radiation spectrum, this equation should be integrated over the photon emitting angles and averaged over directions of velocities of emitting particles, $\boldsymbol{\nu} \equiv \boldsymbol{\beta} /|\boldsymbol{\beta}|$. In order to simplify calculations, let's introduce the following intermediary tensor

$$
\begin{aligned}
T_{\rho \sigma}= & \frac{1}{2} \int\left(\delta_{\rho \sigma}-\nu_{\rho} \nu_{\sigma}\right) \frac{\gamma^{2}\left(1+\gamma^{4} \theta^{4}\right)}{\left(1+\gamma^{2} \theta^{2}\right)^{4}} \\
& \times \delta(\tilde{\omega}+c \boldsymbol{q} \boldsymbol{\nu}-\varkappa) d \Omega d \Omega_{\alpha},
\end{aligned}
$$

where $d \Omega$ and $d \Omega_{\alpha}$ are the solid angles related to the directions of momenta of the emitted photon and the emitting particle, respectively. Note that equation (81) contains all the "directional" terms. Thus, the radiation spectral power can be expressed as

$$
P_{\omega}(t)=\frac{e^{4}}{\pi^{2} m^{2} c^{3}} \int T_{\rho \sigma} \tilde{K}_{\rho \sigma}(\boldsymbol{q}, \varkappa) \frac{d^{3} q}{(2 \pi)^{3}} \frac{d \varkappa}{2 \pi} .
$$

The correlation tensor $K_{\rho \sigma}$ and its Fourier transformation $\tilde{K}_{\rho \sigma}$ are defined in Sec. 3.

According to equation (81), the tensor $T_{\rho \sigma}$ is a symmetric tensor of the second order; it depends only on the vector $\boldsymbol{q}$, therefore, $T_{\rho \sigma}$ has the following structure:

$$
T_{\rho \sigma}=F_{1} \delta_{\rho \sigma}+F_{2} q_{\rho} q_{\sigma}
$$

where $F_{1}$ and $F_{2}$ are scalar functions. The convolution of tensors $T_{\rho \sigma}$ and $\tilde{K}_{\rho \sigma}$, taking into account the transversality condition of equation (21), gives

$$
T_{\rho \sigma} \tilde{K}_{\rho \sigma}=F_{1} \tilde{K}_{\rho \rho},
$$

This expression determine the integrand in equation (82).

To obtain the scalar function $F_{1}$ the following relations can be used:

$$
T_{\rho \rho}=3 F_{1}+q^{2} F_{2}, \quad q_{\rho} q_{\sigma} T_{\rho \sigma}=q^{2} F_{1}+q^{4} F_{2},
$$

which give

$$
F_{1}=\frac{1}{2}\left(T_{\rho \rho}-q_{\rho} q_{\sigma} T_{\rho \sigma} / q^{2}\right) .
$$

Using equation (81), one finds

$$
\begin{gathered}
F_{1}=\frac{1}{4} \int\left(1+\frac{(\boldsymbol{q} \boldsymbol{\nu})^{2}}{q^{2}}\right) \\
\times \frac{\gamma^{2}\left(1+\gamma^{4} \theta^{4}\right)}{\left(1+\gamma^{2} \theta^{2}\right)^{4}} \delta(\tilde{\omega}+c \boldsymbol{q} \boldsymbol{\nu}-\varkappa) d \Omega d \Omega_{\alpha},
\end{gathered}
$$

which after the integration can be presented in the form (see Appendix B)

$$
F_{1}=\frac{\pi^{2}}{3 q c} U(\xi, \varkappa /(q c)) \text {. }
$$

Here $U$ is determined by equation (B5).

The trace of the correlation tensor can be represented as

$$
\tilde{K}_{\rho \rho}(\boldsymbol{q}, \varkappa)=\left\langle\boldsymbol{B}^{2}\right\rangle \Psi(\boldsymbol{q}, \varkappa),
$$

where $\Psi(\boldsymbol{q}, \varkappa)$ satisfies the normalization condition:

$$
\int \Psi(\boldsymbol{q}, \varkappa) \frac{d^{3} q}{(2 \pi)^{3}} \frac{d \varkappa}{2 \pi}=1 .
$$

Thus, the radiation power can be represented as

$$
P_{\omega}(t)=\frac{e^{4}\left\langle\boldsymbol{B}^{2}\right\rangle}{3 m^{2} c^{4}} \int \frac{1}{q} U(\xi, \varkappa / q c) \Psi(\boldsymbol{q}, \varkappa) \frac{d^{3} q}{(2 \pi)^{3}} \frac{d \varkappa}{2 \pi} .
$$

If the correlation function $K_{\rho \sigma}(\boldsymbol{r}, t)$ does not depend on time, i.e. $\Psi(\boldsymbol{q}, \varkappa)=2 \pi \delta(\varkappa) \Psi(\boldsymbol{q})$, then the integration over $\varkappa$ is trivial leading to

$$
P_{\omega}(t)=\frac{e^{4}\left\langle\boldsymbol{B}^{2}\right\rangle}{24 \pi^{3} m^{2} c^{4}} \int u(\xi) \Psi(\boldsymbol{q}) \Theta(\xi-1) \frac{d^{3} q}{q} .
$$

In the derivation of this equation we took into account that

$$
U(\xi, 0)=u(\xi) \Theta(\xi-1) .
$$

Here $u(\xi)$ is defined by equation (32), and $\Theta(x)$ is the Heaviside step function (i.e., $\Theta(x)=1$ if $x>0$ and $\Theta(x)=0$ if $x<0)$.

Obviously, in the case of isotropic turbulence, the general equation (92) should coincide with equation (31). Moreover, equation (31) can describe even the case of anisotropic turbulence, if one substitute the function $\Psi(q)$ by the spectrum of turbulence averaged over directions of the vector $\boldsymbol{q}, \Psi(q) \equiv\langle\Psi(\boldsymbol{q})\rangle$. This implies that the averaged radiation power does not depend on the structure of the correlation tensor. In particular, the monotonic decrease of the intensity given by equation (35), also is observed in the case of anisotropic turbulence.

Although this conclusion is derived under the assumption of isotropic distribution of emitting particles, in fact the obtained result is valid also for the case of anisotropic particle distribution when the change of particle density is small for typical "angular shifts" of the value of $1 / \gamma$. Thus, as long as the emission is considered in the ultrarelativistic regime, this assumption can be broken only in the case of highly collimated particle beams (which is 
likely inconsistent with the underlining assumption of the turbulent magnetic field). Also we note that if we deal with strongly anisotropic distribution of particles, the radiation does depend on the structure of the correlation tensor, therefore it is important to define it correctly (see Section 7).

\section{COMPARISON WITH PREVIOUS RESULTS}

In recent years, a large number of studies have been devoted to calculations of radiation (the magnetic bremsstrahlung) generated by charged particles in smallscale turbulent magnetic fields. However, to our knowledge, the general expression for the radiation spectrum described by equation (31), is derived for the first time in this paper. Also, in the previous studies a few additional conditions have been assumed, which however appear redundant, and actually not needed at all in the framework of our approach. This redundancy not only superficially constraints the applicability of the obtained results, but also introduces some confusion in the analysis and comparison of different radiation regimes. Finally, some solutions and related conclusions derived in this paper do not coincide with the results of previous studies. Therefore, we present below a short overview of a few important papers on the topic, compare their main results with our study, and outline the key differences between the approaches which might cause, in our view, these discrepancies.

There are two basic theoretical approaches to study radiation in random magnetic fields. The first one is based on the seminal paper by TF87, where a kinetic equation has been derived for the probability of different particle trajectories in a chaotic magnetic field (see Eq.(12) TF87), and an approximate solution has been found to this equation (see also Fleishman 2005, for a simplified description of the approach of [F87]. However, the introduced simplifications significantly limit the applicability of this apprbach and do not allow a self-consistent testament of the problem. More specifically, we discuss these issues bellow.

The second approach is based on the perturbation theory (M00; Medvedev 2006; Fleishman 2006). In all these papers, the authors start from an expression for the emission produced by a particle deflected by a small angle in a magnetic field localized in a compact region of space (see Landau \& Lifshitz 1975, § 77). This expression can be written as

$$
\frac{d \mathscr{E}_{\omega}}{d \omega}=\frac{e^{2} \omega}{2 \pi c^{3}} \int_{\omega /\left(2 \gamma^{2}\right)}^{\infty} \frac{\left|\boldsymbol{a}_{\omega^{\prime}}\right|^{2}}{\omega^{\prime 2}}\left(1-\frac{\omega}{\omega^{\prime} \gamma^{2}}+\frac{\omega^{2}}{2 \omega^{\prime 2} \gamma^{4}}\right) d \omega^{\prime}
$$

where $\boldsymbol{a}_{\omega^{\prime}}=\int_{-\infty}^{\infty} \boldsymbol{a}(t) e^{i \omega^{\prime} t} d t$ is the Fourier component of acceleration. However, if the magnetic field occupies a large volume, then even in the case of chaotic magnetic field, the particle deflection will be (unavoidably) large (because of multiple scatterings over emission correlation length). Therefore, the solution based on this expression has a rather limited applicability compared to the practical realizations in the chaotic magnetic field.

The approu employ in our sty also is based on the perturbation theory but it is valid when the particle deflection is small on the typical magnetic field correla- tion length, or, equivalently, if $R_{\mathrm{L}} \gg \lambda$.

Note that the later approximation was also implicitly used in TF87 (see Eq.(11) TF87) when deriving the kinetic equation. So even in the case of precise solution of this equation, the results cannot be expanded beyond the parameter region described by the perturbation theory approach presented in our paper. Moreover, since the derived kinetic equation appeared to be too complex to be treated analytically, a few further simplifications have been introduced to obtain an analytical solution. In particular, the original Eq.(12) TF87 was replaced by Eq.(17) TF87, which indeed could be equivalent to the original one if in the rhs of this equation they would use the function $q(\omega, \theta)$ determined by Eq.(15) TF87. In Eq.(17) TF87 $\omega$ enters as a parameter, thus for solution of this equation it can be taken as a constant, and the function $q$ treated as a function of one variable $q(\theta)$. However, since in this case the equation does not have a solution, the authors replace the function $q(\omega, \theta)$ by an empirical function $q(\omega)$. This simplification allows an analytical solution, but since it concerns the term with the highest derivative in the equation, the uncertainties imposed by this substitution cannot be evaluated and correspondingly, the limits of applicability remain highly unknown. Note that the empirical function $q(\omega)$ itself determines the radiation spectrum in the case of absence of the regular component of the magnetic field. However, within the framework of theory of TF87 this function, strictly speaking, is not derived. Instead, based on arguments of the asymptotic behavior, they have proposed the following form $q(\omega)=q\left(\omega, \theta=\theta_{*}\right)$, where $\theta_{*}^{2}=(a-1) / \gamma^{2}$ and the value of parameter $a$ was determined "from the requirement that at high frequencies, where the perturbation expansion (the method of equivalent photons, see Appendix) is valid, the present method yields the same result as the perturbation expansion".

Let's consider now the results of Fleishman \& Bietenholy (2007), where the approach of TF87 has been applied to the case of the random magnetic field without a regular component. To make the comparison transparent and less bulky, discuss the results for the fix value of the index of the turbulence spectrum $\alpha=2$, and ignore the impact of the surrounding medium (i.e., assume $\left.\omega_{p}=0\right)$. For this specific case, the spectrum obtained in Fleishman \& Bietenholz (2007), can be expressed as:

$$
P_{\omega}=\frac{8 e^{2} \gamma^{2}}{3 \pi c} q(\omega) \Phi(s) .
$$

Here

$$
\begin{gathered}
q(\omega)=\frac{\omega_{s t}^{2} \omega_{0} \gamma^{2}}{(a \omega / 2)^{2}+\left(\omega_{0} \gamma^{2}\right)^{2}}, \\
\Phi(s)=24 s^{2} \int_{0}^{\infty} d t e^{-2 s t} \sin (2 s t)\left(\operatorname{coth} t-\frac{1}{t}\right),
\end{gathered}
$$

where

$$
s=\frac{1}{8 \gamma^{2}}\left(\frac{\omega}{q(\omega)}\right)^{1 / 2}, \quad \omega_{s t}=\frac{c}{R_{L}}, \quad \omega_{0}=\frac{c}{\lambda} .
$$

$\Phi(s)$ has the following asymptotic limits (Fleishman \& Bietenhold 2007):

$$
\Phi(s) \approx 1, \text { if } s \gg 1 ; \quad \Phi(s) \approx 6 s, \text { if } s \ll 1 .
$$


At $\omega \sim \omega_{j}=\omega_{0} \gamma^{2}$ the $s$ parameter is large, $s \sim$ $\omega_{0} / \omega_{s l}=R_{L} / \lambda \gg 1$. Thus one can use the asymptotic limit for $\Phi(s)=1$. Then equation (95) can be expressed in a simple form

$$
P_{\omega}=\frac{8 e^{2} \gamma^{2}}{3 \pi c} q(\omega)
$$

Apparently, the function $q(\omega)$ determines the shape of the radiation spectrum. However, this function has not be derived either by Fleishman \& Bietenholz (2007) or by Fleishman (2005). We can only guess that the authors have used the simplified form of Eq.(39) TF87 (after removal of the bulky complex term from that equation).

Fleishman \& Bietenholz (2007) performed numerical calculation of the radiation spectra also for the case of strong random magnetic field, i.e. in the regime of $\lambda \gtrsim R_{L}$. In the asymptotic limit of $\lambda \gg R_{L}$, the spectrum can be obtained analytically; in this regime we deal with the standard synchrotron spectrum described by equation (55). On the other hand, equation (99) with the asymptotic limit of $\Psi(s)$ for $s \ll 1$ from equation (99), differs significantly from equation (55). In our view, the reason for this discrepancy is that the basic kinetic equation in the theory of TF87 is derived under assumption of $\lambda \ll R_{L}$ (see Eq.(9) TF87). Thus, this approach cannot be applied for the regime $\lambda \geq R_{\mathrm{L}}$.

The fact that equation (95) is not applicable for the case of $\lambda \gg R_{L}$ can be also illustrated by computation of the total power emitted by a particle. Let's consider the ratio of the radiated and lost energies by the relativistic charged particle:

$$
\rho=\int_{0}^{\infty} P(\omega) d \omega / I .
$$

Here $I$ and $P_{\omega}$ are determined by equations (49) and (95), respectively. For a particle emitting in vacuum, the condition $\rho=1$ should be satisfied. In the asymptotic case of $\lambda \ll R_{L}$, one can use equation (100) and demonstrate that for $a=2$ we indeed have $\rho=1$. However, in the limit of $\lambda \gg R_{L}$,

$$
\rho=5.6 \times p^{2 / 3},
$$

where

$$
p=\frac{1}{4} \sqrt{\frac{3}{2 a}} \frac{\omega_{0}}{\omega_{s t}}=\frac{1}{4} \sqrt{\frac{3}{2 a}} \frac{R_{L}}{\lambda} \ll 1,
$$

i.e. the condition $\rho=1$ is violated.

Thus, we can conclude that in the case when the nonchaotic magnetic field is nil, the approached developed by TF87 has a very limited applicability. Namely, one can derive the spectrum in the form of equation (100) with function $q(\omega)$ constrained by asymptotic behavior only.

Now let's compare our results with the studies based, like our paper, on the perturbation theory.

In M00, a specific geometry of interaction has been postulated. Namely, it was assumed that the particle moves along axis $x$, and that magnetic field has only $y$ component, $B_{y}$. Therefore, acceleration is parallel to $z$ axis: $a(t)=\frac{e}{m \gamma} B_{y}(v t, 0,0) \equiv \frac{e}{m \gamma} B(t)$. Although the radiation power obtained in Sect. 3 was derived under the assumption of homogeneity of turbulence, and thus is not applicable to the case considered by M00, it is straightforward to apply our approach to this case also. Namely, accepting the definition of the spectral power given by equation (4), one can average over the magnetic field configurations in equation (94). This gives

$$
\begin{gathered}
P_{\omega}(t)=\frac{e^{4} \omega}{2 \pi m^{2} \gamma^{2} c^{3}} \\
\times \int_{\omega /\left(2 \gamma^{2}\right)}^{\infty} \frac{\left(\tilde{B}^{2}\right) \omega^{\prime}}{\omega^{\prime 2}}\left(1-\frac{\omega}{\omega^{\prime} \gamma^{2}}+\frac{\omega^{2}}{2 \omega^{\prime 2} \gamma^{4}}\right) d \omega^{\prime}
\end{gathered}
$$

where

$$
\left(\tilde{B}^{2}\right)_{\omega^{\prime}} \equiv \int_{-\infty}^{\infty}\langle B(t+\tau / 2) B(t-\tau / 2)\rangle e^{i \omega^{\prime} \tau} d \tau .
$$

is the Fourier component of the magnetic field correlation function; generally it may depend not only on $\omega^{\prime}$, but also on $t$. Obviously,

$$
\left\langle B^{2}(t)\right\rangle=\int_{0}^{\infty}\left(\tilde{B}^{2}\right)_{\omega} \frac{d \omega}{\pi} .
$$

Equation (104) describes the radiation power for the geometry postulated by for an arbitrary spectrum of turbulence. Then, a specific spectrum of turbulence has been considered in M00 for derivation of Eq.(17) $)_{\text {M00 }}$. The interesting feature of the latter is that in the limit of $\omega \rightarrow 0$ the spectrum $P_{\omega} \sim \omega$, and contains an abrupt cutoff, $P_{\omega}=0$ at $\omega>2 \omega_{j}$. However, we should note that these spectral features do not characterize the jitter radiation in general (as it can be seen from equation (104)), but simply are the consequence of the choice of a specific turbulence spectrum and/or interaction geometry (see also Fleishman 2006). Indeed, if one adopts a different turbulence spectrum, e.g. $\left(\tilde{B}^{2}\right)_{\omega^{\prime}} \sim\left(\omega^{\prime 2}+\omega_{*}^{2}\right)^{-\alpha}$, then for any positive value of the index $\alpha$, the spectrum is a monotonically decreasing function of $\omega$. Moreover, if the stochastic field has both $y$ - and $z$-components, and the correlation function is azimuthally symmetric in respect to $x$-axis, then even for the power-law spectrum of turbulence, adopted by M00, the spectrum is not expected to be linear in the limit of small $\omega$.

The treatment of radiation in a chaotic magnetic field always leads to the appearance of the correlation tensor, $\tilde{K}_{\rho \sigma}$ (see equation (19) of this paper and Eq.(12) of Eleishman 2006). However, often the structure of this tensor, $\bar{K}_{\rho \sigma}(\boldsymbol{q}, \varkappa)$, is wrongly postulated. If we consider a homogeneous environment without preferred directions, then we deal with only two second-order tensors: $\delta_{\rho \sigma}$ and $q_{\rho} q_{\sigma}$. Therefore, the correlation tensor should have the following structure: $\tilde{K}_{\rho \sigma}=c_{1} \delta_{\rho \sigma}+c_{2} q_{\rho} q_{\sigma}$, where $c_{1,2}$ are two scalar functions. The transversality condition implies $c_{1}+c_{2} q^{2}=0$, thus $\tilde{K}_{\rho \sigma}$ has to be proportional to $\left(\delta_{\rho \sigma}-q_{\rho} q_{\sigma} / q^{2}\right)$, as used in equation (22). However, in the case of existence of a distinct direction, $s$, e.g. normal to the shock front, the tensor structure 
becomes more complex:

$$
\tilde{K}_{\rho \sigma}=c_{1} \delta_{\rho \sigma}+c_{2} q_{\rho} q_{\sigma}+c_{3} s_{\rho} s_{\sigma}+c_{4} q_{\rho} s_{\sigma}+c_{5} s_{\rho} q_{\sigma} .
$$

The transversality condition imposes three constraints on the scalar functions $c_{i}$, thus the correlation tensor $\tilde{K}_{\rho \sigma}$ must have the following structure:

$$
\begin{gathered}
\tilde{K}_{\rho \sigma}=\Psi_{1}\left(\delta_{\rho \sigma}-\frac{q_{\rho} q_{\sigma}}{q^{2}}\right) \\
+\Psi_{2}\left(s_{\rho}-q_{\rho} \frac{(\boldsymbol{s q})}{q^{2}}\right)\left(s_{\sigma}-q_{\sigma} \frac{(\boldsymbol{s q})}{q^{2}}\right),
\end{gathered}
$$

where functions $\Psi_{1,2}$ depend on three arguments: $|\boldsymbol{q}|$, $(\boldsymbol{s q})$ and $\varkappa$. In a gyrotropic medium, the correlation function may contain an additional term: $\epsilon_{\rho \sigma \tau} q_{\tau} \Psi_{3}$, where $\epsilon_{\rho \sigma \tau}$ is the Levi-Civita-tensor and $\Psi_{3}$ is a complex function as it follows from the general theory of fluctuations (see e.g. Landau \& Lifshitz 1980, § 122). Note, however, that this term does not contribute to the emission power, since in equation (24) the tensor $\tilde{K}_{\rho \sigma}$ is convolved with a symmetric tensor.

For the additional assumption that the magnetic field is perpendicular to the direction $s$, the correlation function should satisfy the equations $\tilde{K}_{\rho \sigma} s_{\rho}=0, \tilde{K}_{\rho \sigma} s_{\sigma}=0$. In this case the functions $\Psi_{1}$ are linked $\Psi_{2}$ via the relation

$$
\Psi_{1}+\left(1-(\boldsymbol{s q})^{2} / q^{2}\right) \Psi_{2}=0
$$

and the correlation function $\tilde{K}_{\rho \sigma}$ is determined just by one scalar function.

However, in some studies dealing with the anisotropic turbulence, different tensor structures have been proposed for the correlation function: $\tilde{K}_{\rho \sigma} \propto\left(\delta_{\rho \sigma}-s_{\rho} s_{\sigma}\right)$ - we can refer, for example to Eq.(8) in Medvedev 2006. Eq.(18) in Fleishman 2006, Eq.(10) in Medvedev et al. 2011, Eq.(11) in Reynolds \& Medvedev 2012). This correlation function does not satisfy the transversality condition, i.e., the considered magnetic field is not divergence free, $\nabla \boldsymbol{B} \neq 0$. Apparently, this is a wrong result, therefore the results obtained under the assumption of $\tilde{K}_{\rho \sigma} \propto\left(\delta_{\rho \sigma}-s_{\rho} s_{\sigma}\right)$ should be revised.

It is important to note that certain mathematical operation often used for computation of emission in chaotic magnetic field lack mathematical strictness (also see discussions in Fleishman 2006; Medvedev 2005). In particular, this concerns the involvement of the field harmonics, $B_{k}$, which implies that the Fourier transformation can be applied to the stochastic magnetic field. This assumption hardly can be justified or disproved from the first principles, however this assumption may lead to a rather controversial expression for the Fourier image of the correlation function. For example, the following structure has been obtained for the correlation tensor $\tilde{K}_{\rho \sigma} \propto \tilde{B}_{0} \tilde{B}_{\sigma}$ (see footnote 2 and equation 5 in Fleishman 2006, Medvedev 2006, respectively), which, however, contradicts the general tensor structure given by equation (108) (see also Landau \& Lifshitz 1980, § 122).

Finally, we note that in the framework of our approach, no assumptions regarding the properties of the stochastic magnetic field are required. Instead, we assumed that the Fourier transformation can be applied to the magnetic field correlation function, which is a significantly less demanding assumption.

\section{DISCUSSION AND SUMMARY}

The so-called jitter radiation mechanism represents a version of the magnetic bremsstrahlung of relativistic charged particles in a turbulent magnetic field. This regime of radiation can be realized with an efficiency as high as the "standard" synchrotron radiation but with quite distinct energy spectrum strongly shifted towards higher energies. This makes the jitter radiation an attractive gamma-ray production channel in highly turbulent astrophysical environments.

In this paper we present a novel study on spectral properties of the jitter radiation performed within the framework of perturbation theory in the regime of the so-called small-scale turbulence, when the coherent length of the field is significantly smaller than the nonrelativistic Larmor radius, $\lambda \ll R_{L}=m c^{2} / e B$, or

$$
\lambda \ll 1.7 \times 10^{3}\left(\mathrm{~m} / \mathrm{m}_{e}\right)(B / 1 \mathrm{G})^{-1} \mathrm{~cm} .
$$

Here $B$ is the average magnetic field, and $m$ is the mass of radiating charged particle. It is remarkable that the condition for realization of the jitter regime does not depend on the particle energy, but only on its mass. For example, for electrons the condition imposed by equation (110) implies a turbulence scale as small as $100 \mathrm{~km}$ in young supernova remnants, less than $10 \mathrm{~m}$ in gammaray production regions of blazers, and $1 \mathrm{~cm}$ in GRBs, assuming typical values of the magnetic field in these objects of about $100 \mu \mathrm{G}, 1 \mathrm{G}$ and $1 \mathrm{kG}$, respectively. For protons these conditions are relaxed by three orders of magnitude. However, the magnetic bremsstrahlung of protons is a much slower process compared to electrons. It becomes adequately effective only at very high energies of protons and at the presence of large magnetic field which in its turn implies tighter conditions on the turbulence scale. Whether turbulent fields can be generated at scales imposed by equation (110) is a non-trivial issue the discussion of which is beyond the scope of this paper. Here we focused merely on the study of radiation properties and perform calculations under the assumption that equation (110) is (by definition) fulfilled. We derived an expression for the spectral power of radiation presented in a general but rather compact form convenient for numerical calculations.

The jitter radiation has a simple spectral form. Its SED for a monoenergetic particle distribution is shown in Fig. 1 together with the SED of synchrotron radiation. Both SEDs have pronounced maximums separated from each other by a factor of $R_{L} / \lambda$. Obviously when the jitter regime is realized, the maximum of its SED is shifted towards higher energies (the position of the peak in the synchrotron SED is at the energy $\left.\approx 1.115 \omega_{c}\right)$. Unless one introduces strong assumptions regarding the turbulence spectrum and/or its geometry, the low energy part of the spectrum has standard photon index $\Gamma=1$. It is hard but still softer than the spectrum of synchrotron radiation with $\Gamma=4 / 3$. The jitter and synchrotron spectra are very different beyond their respective maximums. While the standard synchrotron spectrum cuts off quite sharply (exponentially) just after the maximum, the spectrum of the jitter radiation continues as a powerlaw until the energy $\sim\left(R_{L} / \lambda\right)^{3} \omega_{c}$ with a photon index $\Gamma=\alpha+1$, where $\alpha$ is the power-law index of the turbulence spectrum (in the framework of the perturbation 
theory, the spectral shape of radiation above this limit cannot be calculated). Remarkably, this part of the spectrum is not sensitive to the details of the energy distribution of particles, but depends only on the position of the cutoff in the particles distribution. The latter determines the start of the power-law tail which should be (by definition) quite long since $R_{L} \gg \lambda$. For example, if the ratio $R_{L} / \lambda$ exceeds 10 , the power-law tail of the jitter radiation, which mimic the turbulence spectrum, would span over more than two energy decades after the maximum. Bellow the maximum, the jitter radiation does depend on the particle distribution. In particular, if the relativistic particles have a power-law distribution with an index $\mu$, the spectrum of the jitter radiation is also power-law with photon index $\Gamma=(\mu+1) / 2$, i.e. exactly the same as in the case of synchrotron radiation.

In this paper we do not aim to discuss astrophysical implications of jitter radiation which deserve separate consideration. Instead, we would rather comment on some interesting features which make this mechanism quite unique amongst other high energy radiation processes.

First of all, the slight dependence (or rather independence) of the high energy spectral tail on the distribution of parent relativistic particles, is quite unusual and apparently does not have an analog in astrophysics. Moreover, the fact that the spectral shape of this tail simply mimics the spectrum of turbulence, opens a unique opportunity for the straightforward probe of the spectrum of small-scale turbulence by measuring the characteristic high energy electromagnetic radiation and identifying it with the jitter mechanism.

While in the case of injection of relativistic electrons into a highly turbulent medium, where the condition of equation (110) is satisfied, guaranties production of radiation in the jitter regime, the questions of its detection depends on the total energetics in relativistic particles. Given the usually (very) high efficiency of jitter radiation, and for typical parameters characterizing the nonthermal astrophysical sources of both galactic and extragalactic origin, the production of detectable fluxes of jitter radiation in the X-ray and/or gamma-ray bands could be readily realized in standard acceleration and radiation scenarios.

The identification of the origin or radiation is the second critical issue. Fortunately, the jitter radiation does provide us with distinct features which can be used to identify its nature. In particular, for a standard powerlaw injection distribution of electrons with a high energy cutoff given, for example, in the form of equation (77) with $\mu=2$, and assuming a Kolmogorovtype spectrum of turbulence, $\alpha=5 / 3$, we expect a gamma-ray spectrum which can be described as broken power-law. The high energy part of the spectrum above the break is expected to have a photon index of $\Gamma_{2}=\alpha+1 \simeq 2.7$, while the low energy part (below the break) $\Gamma_{1}=(\mu+1) / 2=1.5$ or $\Gamma_{1}=(\mu+2) / 2=2$ for the slow and fast cooling regimes, respectively. This corresponds to the change of the spectral index by $\Delta \Gamma=1.2$ or 0.7 depending on the cooling regime. Such a behavior differs significantly from the standard synchrotron cooling break with $\Delta \Gamma=0.5$. In the case of a low energy cutoff in the electron spectrum, which is often required to fit the data, e.g. the spectra of gamma-ray blazars, we should expect another break below which the photon index would be $\Gamma=1$ Therefore, in the case of detection of a non-standard broken power-law spectra, especially when the high energy power-law tail has a photon index close to 2.5 and extends well beyond the break, the jitter mechanism can be be treated as a process responsible for the observed spectral features (see also Fleishman \& Bietenhold 2007).

Despite all attractive properties of synchrotron radiation of ultrarelativistic electron, its spectrum usually terminates before reaching the gamma-ray domain. Even in the extreme accelerators it cannot extend beyond the socalled synchrotron limit $\sim 100 \mathrm{MeV}$, unless being additionally Doppler boosted in sources with relativistic Doppler factors. This can be the case, for example, of the recently discovered flares of the Crab Nebula (see, e.g. Buehler et al. 2012; Striani et al. 2013, and references therein) or the multi-GeV counterparts of gammaray bursts (Abdo et al. 2009). On the other hand, the jitter mechanism may offer another possibility for extension of the spectrum well beyond the energy synchrotron limit. We should note in this regard that in the case of fulfillment of the condition in equation (110), the appearance of jitter radiation is not only unavoidable, but its spectrum could extend to high or even very high energies. A more a principal issue in this regard is the challenge of formation of turbulence on very small scales, e.g. through the Weibel type instabilities.

APPENDIX

\section{HIGH ENERGY ASYMPTOTICS OF SYNCHROTRON RADIATION}

At high frequencies, the intensity of synchrotron radiation decreases exponentially, $G(x) \propto \exp \left(-\frac{2 \omega m c}{3 e B \gamma^{2}}\right)=$ $\exp \left(-\frac{\omega}{b \omega_{0}}\right)$ (see equation (58)). Therefore at $\omega \gg \omega_{0}$ the contribution to the radiation spectrum given by equation (66) is dominated by regions characterized by large magnetic field: $b \gg 1$. Let us assumed that for $b \gg 1$, the magnetic field strength is distributed as power-law : $w d B \approx A b^{-\sigma} d b$.

It is convenient to start the calculations of the spectrum from equation (53), where magnetic field strength is replaced by $B \rightarrow B \sin \chi$, i.e. before integration over directions of the magnetic field. This gives

$$
P_{\omega}=\frac{\sqrt{3} e^{2}}{2 \pi R_{L 0}} x \int_{x / b \sin \chi}^{\infty} K_{5 / 3}(u) d u,
$$

where $x=\omega / \omega_{0}, R_{L 0}=m c^{2} / e B_{0}$ and $b=B / B_{0}$. To average over the strength of the magnetic field strength, 
one should multiply equation (A1) to the distribution of magnetic field, $A b^{-\sigma} d b$, and then integrate over $d b$. Let us introduce a new variable $b \rightarrow \xi=x / b \sin \chi$, and change the order of integration over $d \xi$ and $d u$. Then, after a rather simple analytical integration over $d \xi$, we obtain:

$$
\int P_{\omega} w(b) d b=A \frac{\sqrt{3} e^{2}}{2 \pi R_{L 0}} \frac{x^{-\sigma+2}(\sin \chi)^{\sigma-1}}{\sigma-1} \int_{0}^{\infty} u^{\sigma-1} K_{5 / 3}(u) d u .
$$

The remaining integral over $d u$ can be expressed in terms of Gamma functions:

$$
\int_{0}^{\infty} u^{\sigma-1} K_{5 / 3}(u) d u=2^{\sigma-2} \Gamma\left(\frac{\sigma}{2}+\frac{5}{6}\right) \Gamma\left(\frac{\sigma}{2}-\frac{5}{6}\right) .
$$

The integration over pitch-angles also leads to an expression containing Gamma functions:

$$
\left\langle(\sin \chi)^{\sigma-1}\right\rangle=\frac{\sqrt{\pi} \Gamma\left(\frac{\sigma}{2}+\frac{1}{2}\right)}{2 \Gamma\left(\frac{\sigma}{2}+1\right)} .
$$

Thus, in the limit of large frequency $\omega \gg \omega_{0}$, the spectrum of synchrotron radiation averaged over the directions and strength of the magnetic field, is described by a power-law function:

$$
\left\langle P_{\omega}\right\rangle=A \frac{e^{2}}{R_{L 0}} C_{\sigma} x^{-\sigma+2},
$$

where

$$
C_{\sigma}=\frac{\sqrt{3} 2^{\sigma-4}}{\sqrt{\pi}(\sigma-1)} \frac{\Gamma\left(\frac{\sigma}{2}+\frac{5}{6}\right) \Gamma\left(\frac{\sigma}{2}-\frac{5}{6}\right) \Gamma\left(\frac{\sigma}{2}+\frac{1}{2}\right)}{\Gamma\left(\frac{\sigma}{2}+1\right)} .
$$

Note that if the magnetic filed is formally distributed as pure power-law, $B: w(B)=\bar{A} B^{-\sigma}$, equation (A5) gives precise solution for the radiation spectrum. In this case $A=\bar{A} B_{0}^{1-\sigma}$, and therefore $\left\langle P_{\omega}\right\rangle$ appears to be independent of $B_{0}$.

To understand the condition for applicability of equation ( $\mathrm{A} 5)$, let us estimate the correction terms to this equation for a specific distributions of the magnetic field. Let's assume, for example, the following distribution:

$$
w(B) d B=\frac{A b^{2}}{\left(1+b^{2}\right)^{1+\sigma / 2}} .
$$

In the limit $b \gg 1$, the first two terms of the series are

$$
\frac{A b^{2}}{\left(1+b^{2}\right)^{1+\sigma / 2}} \approx A\left(\frac{1}{b^{\sigma}}-\frac{1+\sigma / 2}{b^{\sigma+2}}\right) .
$$

Correspondingly,

$$
\left\langle P_{\omega}\right\rangle=A \frac{e^{2}}{R_{L 0}}\left(C_{\sigma} x^{-\sigma+2}-(1+\sigma / 2) C_{\sigma+2} x^{-\sigma}\right) .
$$

The ratio of these two terms can be expressed as

$$
r=\frac{(\sigma-1)\left(9 \sigma^{2}-25\right)}{18 x^{2}} \sim \frac{\sigma^{3}}{2 x^{2}},
$$

which implies that one can neglect the second term when $x \gtrsim \sigma^{3 / 2}$. This can be treated as the condition for applicability of equation (A5).

\section{THE CASE OF ANISOTROPIC TURBULENCE}

Here we present some intermediate calculations required for derivation of equation (88) from equation (87). To compute the integrals, it is convenient to introduce the following new variables:

$$
\zeta=\gamma^{2} \theta^{2}, \quad x=\cos \vartheta,
$$

where $\vartheta$ is the angle between vectors $\boldsymbol{\nu}$ and $\boldsymbol{q}$. The integration over the azimuthal angle is trivial; it gives $d \Omega d \Omega_{\alpha}=$ $\frac{2 \pi^{2}}{\gamma^{2}} d x d \zeta$. Then, the integration of equation (87) results in

$$
F_{1}=\frac{\pi^{2}}{2} \int_{0}^{\infty} d \zeta \int_{-1}^{1} d x\left(1+x^{2}\right) \frac{1+\zeta^{2}}{(1+\zeta)^{4}} \delta(\tilde{\omega}+c q x-\varkappa) .
$$


For the upper limit of integration over $\zeta$ we set $\infty$, which is valid only in the ultrarelativistic regime (see also the discussion after equation (30)). The argument of the $\delta$-function nulls for $x=x_{0}=(\varkappa-\tilde{\omega}) / c q$. The integral becomes zero if $x_{0}$ lies beyond the integration interval, $x_{0}>1$ or $x_{0}<-1$. For the value of $x_{0}$ within the integration range, i.e., $\left|x_{0}\right|<1$, we obtain

$$
F_{1}=\frac{\pi^{2}}{2 c q} \int_{\zeta_{1}}^{\zeta_{2}} d \zeta\left(1+x_{0}^{2}\right) \frac{1+\zeta^{2}}{(1+\zeta)^{4}}
$$

where the lower and upper integration limits, $\zeta_{1,2}$, are determined by the conditions $\left|x_{0}\right|=1$ and $\zeta \geq 0$.

It is convenient to express these limits as $\zeta_{1}=\max (0, \xi(\kappa-1)-1)$ and $\zeta_{2}=\xi(\kappa+1)-1\left(\zeta_{2}\right.$ should be positive), where

$$
\kappa=\frac{\varkappa}{q c}, \quad \xi=\frac{2 q c \gamma^{2}}{\omega} .
$$

This allows derivation of equation (88) via the analytical integration:

$$
U(\xi, \kappa)=\Theta(\xi(\kappa+1)-1)\left[U_{1} \Theta(1-\xi(\kappa-1))+U_{2} \Theta(\xi(\kappa-1)-1)\right]
$$

where

$$
U_{1}=\frac{1}{\xi^{3}}(\xi(\kappa+1)-1)\left(\xi^{2}(\kappa-1)+4 \xi+\frac{2 \xi^{2}-2 \xi+1}{\kappa+1}-\frac{\xi-1}{(\kappa+1)^{2}}+\frac{2}{(\kappa+1)^{3}}\right)-\frac{3}{\xi^{2}}(\xi \kappa+1) \ln (\xi(\kappa+1)),
$$

and

$$
U_{2}=\frac{2}{\xi^{3}\left(\kappa^{2}-1\right)^{3}}\left(4+3 \kappa^{6} \xi^{2}+3 \xi \kappa^{5}-6 \xi^{2} \kappa^{4}-12 \xi \kappa^{3}+\left(3 \xi^{2}+4\right) \kappa^{2}+9 \xi \kappa\right)-\frac{3}{\xi^{2}}(\xi \kappa+1) \ln \left(\frac{\kappa+1}{\kappa-1}\right) .
$$

Equation (B5) implies that function $U(\xi, \kappa)$ has non-zero values only if $\kappa>\frac{1}{\xi}-1$. The two terms in equation (B5), $U_{1,2}$, give non-zero contribution for $\frac{1}{\xi}-1<\kappa<\frac{1}{\xi}+1$ and $\kappa>\frac{1}{\xi}+1$, respectively. The continues function $U$ has a break at $\kappa=\frac{1}{\xi}+1 U(\xi, \kappa)$.

\section{ACKNOWLEDGMENTS}

We would like to thank A. Bykov, G. Fleishman, B. Reville and Y. Teraki for helpful discussions. We should also mention that during our communications G. Fleishman informed us that some of our critical comments from Sect. of this paper concerning the previous works he had discussed in his book "Stohasticheskaja teorija izluchenija" (in Russian, 2008, ISBN 978-5-93972-624-5).

\section{REFERENCES}

Abdo, A. A., et al. 2009, Science, 323, 1688

Aharonian, F. A., Belyanin, A. A., Derishev, E. V., Kocharovsky, V. V., \& Kocharovsky, V. V. 2002, Phys. Rev. D, 66, 023005 Aharonian, F. A., Kelner, S. R., \& Prosekin, A. Y. 2010, Phys. Rev. D, 82, 043002

Berestetskii, V. B., Lifshitz, E. M., \& Pitaevskii, L. P. 1989, Quantum Electrodynamics (Pergamon Press, Oxford)

Buehler, R., Scargle, J. D., Blandford, R. D., et al. 2012, ApJ, 749,26

Bykov, A. M., Pavlov, G. G., Artemyev, A. V., \& Uvarov, Y. A. 2012, MNRAS, 421, L67

Crusius, A., \& Schlickeiser, R. 1986, A\&A, 164, L16

Derishev, E. V. 2007, Ap\&SS, 309, 157

Fleishman, G. D. 2005, ArXiv Astrophysics e-prints 0510317

Fleishman, G. D. 2006, ApJ, 638, 348

Fleishman, G. D., \& Bietenholz, M. F. 2007, MNRAS, 376, 625

Fritz, K. D. 1989, A\&A, 214, 14

Ginzburg, V. L., \& Syrovatskii, S. I. 1969, ARA\&A, 7, 375

Iroshnikov, P. S. 1963, AZh, 40, 742

Jackson, J. D. 1998, Classical Electrodynamics (New York: Wiley)

Kirk, J. G., \& Reville, B. 2010, ApJ, 710, L16

Kolmogorov, A. 1941, Akademiia Nauk SSSR Doklady, 30, 301

Kraichnan, R. H. 1965, Physics of Fluids, 8, 1385
Landau, L. D., \& Lifshitz, E. M. 1975, The Classical Theory of Fields (Pergamon Press, Oxford)

—. 1980, Statistical Physics, Part 1 (Pergamon Press, Oxford)

Lefa, E., Kelner, S. R., \& Aharonian, F. A. 2012, ApJ, 753, 176

Mao, J., \& Wang, J. 2007, ApJ, 669, L13

Medvedev, M. V. 2000, ApJ, 540, 704 (M00

-. 2005, ArXiv Astrophysics e-prints 0503463

-. 2006, ApJ, 637, 869

Medvedev, M. V., Trier Frederiksen, J., Haugbølle, T., \&

Nordlund, A. 2011, ApJ, 737, 55

Reynolds, S. J., \& Medvedev, M. V. 2012, Physics of Plasmas, 19, 023106

Reville, B., \& Kirk, J. G. 2010, ApJ, 724, 1283

Rybicki, G. B., \& Lightman, A. P. 1979, Radiative Processes in Asrtophysics (New York: Wiley)

Schwinger, J. 1949, Phys. Rev., 75, 1912

Striani, E., et al. 2013, ApJ, 765, 52

Teraki, Y., \& Takahara, F. 2011, ApJ, 735, L44

Teraki, Y., \& Takahara, F. 2013, ApJ, 763, 131

Toptygin, I. N., \& Fleishman, G. D. 1987, Ap\&SS, 132, 213 (TF87)

Zirakashvili, V. N., \& Aharonian, F. 2007, A\&A, 465, 695 